\title{
Dissecting the genetics of chronic mucus hypersecretion in smokers with and without COPD
}

Akkelies E. Dijkstra, H. Marike Boezen, Maarten van den Berge, Judith M. Vonk, Pieter S. Hiemstra, R. Graham Barr, Kirsten M. Burkart, Ani Manichaikul, Tess D. Pottinger, Edward K. Silverman, Michael H. Cho, James D. Crapo, Terri H. Beaty, Per Bakke, Amund Gulsvik, David A. Lomas, Yohan Bossé, David C. Nickle, Peter D. Paré, Harry J. de Koning, Jan-Willem Lammers, Pieter Zanen, Joanna Smolonska, Ciska Wijmenga, Corry-Anke Brandsma, Harry J.M. Groen, Dirkje S. Postma and the LifeLines Cohort Study group

Affiliation: For lists of the authors' affiliations, and the LifeLines Cohort Study group members and their affiliations, see the Acknowledgements section.

Correspondence: Dirkje S. Postma, University Medical Center Groningen, Dept of Pulmonology, Hanzeplein 1 , 9700 RB Groningen, The Netherlands. E-mail: d.s.postmađumcg.nl

ABSTRACT Smoking is a notorious risk factor for chronic mucus hypersecretion (CMH). CMH frequently occurs in chronic obstructive pulmonary disease (COPD). The question arises whether the same single-nucleotide polymorphisms (SNPs) are related to $\mathrm{CMH}$ in smokers with and without COPD.

We performed two genome-wide association studies of $\mathrm{CMH}$ under an additive genetic model in male heavy smokers $(\geqslant 20$ pack-years) with COPD $(n=849,39.9 \% \mathrm{CMH})$ and without COPD $(\mathrm{n}=1348,25.4 \%$ $\mathrm{CMH}$ ), followed by replication and meta-analysis in comparable populations, and assessment of the functional relevance of significantly associated SNPs.

Genome-wide association analysis of $\mathrm{CMH}$ in COPD and non-COPD subjects yielded no genome-wide significance after replication. In COPD, our top SNP ( $r 10461985, p=5.43 \times 10^{-5}$ ) was located in the GDNF-AS1 gene that is functionally associated with the GDNF gene. Expression of GDNF in bronchial biopsies of COPD patients was significantly associated with $\mathrm{CMH}(\mathrm{p}=0.007)$. In non-COPD subjects, four SNPs had a p-value $<10^{-5}$ in the meta-analysis, including a SNP (rs4863687) in the MAML3 gene, the T-allele showing modest association with $\mathrm{CMH}\left(\mathrm{p}=7.57 \times 10^{-6}\right.$, OR 1.48) and with significantly increased MAML3 expression in lung tissue $\left(\mathrm{p}=2.59 \times 10^{-12}\right)$.

Our data suggest the potential for differential genetic backgrounds of $\mathrm{CMH}$ in individuals with and without COPD.

@ERSpublications

Genetic determinants of chronic mucus hypersecretion may differ by COPD status http://ow.ly/AeqCr 


\section{Introduction}

Chronic mucus hypersecretion $(\mathrm{CMH})$ can be present in individuals with or without chronic obstructive pulmonary disease (COPD). The prevalence of $\mathrm{CMH}$ varies from $3.5 \%$ to $12.7 \%$ in the general population depending on the population studied and the $\mathrm{CMH}$ definition used $[1,2]$. The prevalence of $\mathrm{CMH}$ is much higher in individuals with COPD $(30 \%)$ and increases with the severity of airflow limitation $[3,4]$. Some risk factors for COPD and $\mathrm{CMH}$ overlap, like smoking, occupational exposures and bacterial infections [5-9].

However, not all heavy smokers have $\mathrm{CMH}$, which may be explained by a genetic contribution to $\mathrm{CMH}$, as evidenced by familial aggregation of mucus overproduction and higher concordance of $\mathrm{CMH}$ in monozygotic than in dizygotic twins [10-12]. So far, only two genetic studies on $\mathrm{CMH}$ have been published. One study suggested that the cytotoxic T-lymphocyte-associated protein 4 gene (CTLA4) is associated with chronic bronchitis in individuals with COPD without a direct association with COPD itself [13]. A second study showed that a single-nucleotide polymorphism (SNP) (rs6577641) in the SATB homeobox 1 gene (SATB1) was strongly associated with $\mathrm{CMH}$ in a heavy-smoking population [14].

As not all individuals with COPD have $\mathrm{CMH}$ and, conversely, not all individuals with $\mathrm{CMH}$ have COPD, the question arises whether similar or differential genetic factors are involved in the development of $\mathrm{CMH}$ in individuals with and without COPD. Therefore, we performed a genome-wide association (GWA) study on $\mathrm{CMH}$ in a group of male individuals with COPD and a group without COPD, from the same heavy-smoking, general population-based cohort (NELSON) [15]. Subsequently, we evaluated our findings on the association with $\mathrm{CMH}$ in replication cohorts including individuals with and without COPD, and searched for features of our most significant findings.

\section{Methods}

\section{Ethics statement}

The Dutch Ministry of Health and the Medical Ethics Committee of each hospital approved the study protocol for the Dutch centres. Ethics approval and written informed consent was obtained from all participants in the studies. For detailed information, see the online supplementary material.

\section{Identification population}

Male Caucasian participants from Groningen and Utrecht, the Netherlands, were included from the Dutch NELSON study [15], a heavy-smoking population-based lung cancer screening study. Information on $\mathrm{CMH}$ and smoking behaviour was collected by questionnaires as published previously [14]. Spirometry was performed according to the European Respiratory Society guidelines, including forced expiratory volume in $1 \mathrm{~s}(\mathrm{FEV} 1)$ and forced vital capacity (FVC), without using a bronchodilator [16]. COPD was defined as $\mathrm{FEV} 1 / \mathrm{FVC}<0.70$.

Support statement: The COPACETIC study was funded by European Union (EU) Seventh Framework grant 201379 The NELSON study was supported by Zorg Onderzoek Nederland-Medische Wetenschappen, KWF Kankerbestrijiding, and Stichting Centraal Fonds Reserves van Voormalig Vrijwillige Ziekenfondsverzekeringen. The LifeLines Cohort Study was sponsored by the Dutch ministry of Health, Welfare and Sport, the Ministry of Economic Affairs, Agriculture and Innovation, the Province of Groningen, the EU Regional Development Fund, the Northern Netherlands Provinces (SNN), the Netherlands Organisation for Scientific Research (NWO), University Medical Center Groningen, University of Groningen, de Nierstichting (the Dutch Kidney Foundation) and the Diabetes Fonds (the Diabetic Foundation). The COPDGene study was funded by US National Institutes of Health (NIH) grants R01 HL089856 and R01 HL089897, and by the COPD Foundation through contributions made to an Industry Advisory Board comprised of AstraZeneca, Boehringer Ingelheim, Novartis, Pfizer, and Sunovion. The ECLIPSE study was funded by GlaxoSmithKline. Data sampling for the GenKOLS study was funded by GlaxoSmithKline. The MESA Lung/SHARe Study was funded by NIH grant RC1HL100543. MESA and the MESA SHARe project are conducted and supported by contracts N01-HC-95159 through N01-HC-95169 and RR-024156 from the National Heart, Lung, and Blood Institute (NHLBI). MESA Air is conducted and supported by the US Environmental Protection Agency in collaboration with the MESA Air investigators, with support provided by grant RD83169701. Funding for MESA SHARe genotyping was provided by NHLBI contract N02-HL-6-4278. MESA Family is conducted and supported in collaboration with the MESA investigators; support is provided by grants and contracts R01HL071051, R01HL071205, R01HL071250, R01HL071251, R01HL071252, R01HL071258, R01HL071259, M01-RR00425, UL1RR033176 and UL1TR000124. The MESA Lung and MESA COPD Studies are funded by NIH grants R01HL077612 and R01HL093081. A full list of participating MESA investigators and institutions can be found at www.mesa-nhlbi.org. The lung eQTL study at Laval University was supported by the Chaire de Pneumologie de la Fondation J.D. Bégin de l'Université Laval, the Fondation de l'Institut Universitaire de Cardiologie et de Pneumologie de Québec, the Respiratory Health Network of the Fonds de Recherche Québec - Santé (FRQS), the Canadian Institutes of Health Research (MOP - 123369), the Cancer Research Society, and Read for the Cure. Y. Bossé is the recipient of a Junior 2 Research Scholar award from the FRQS.

Conflict of interest: Disclosures can be found alongside the online version of this article at erj.ersjournals.com 
To assess whether different genetic factors contribute to the presence of $\mathrm{CMH}$ in smoking individuals with and without COPD, we conducted two GWA studies; one in NELSON individuals with COPD (NELSON-COPD) and a second in NELSON participants without COPD (NELSON-non-COPD) [15].

\section{Replication populations}

Top hits associated with CMH in NELSON-COPD were in silico-analysed in individuals with $\geqslant 5$ packyears smoking and FEV1/FVC $<0.70$ from four independent, Caucasian COPD cohorts: GenKOLS, COPDGene, ECLIPSE and MESA [17-20]. Subsequently meta-analyses were performed across these replication cohorts, and across NELSON-COPD and these replication cohorts.

Top hits associated with CMH in NELSON-non-COPD were analysed in the general population cohort LifeLines by selecting individuals without COPD and $\geqslant 5$ pack-years smoking.

A description of the replication cohorts is given in the online supplementary material. Details on the identification and replication cohorts concerning genotyping method, genotyping imputation software, and $\mathrm{CMH}$ and $\mathrm{COPD}$ definitions are given in online supplementary table 1.

\section{Functional relevance of identified top SNPs}

We assessed whether the top SNPs in individuals with and without COPD were associated with gene expression levels in human lungs. Expression quantitative trait loci (eQTLs) were identified in 1095 lung tissues from three independent cohorts recruited from Laval University (Québec City, QC, Canada), the University of British Columbia (Vancouver, BC, USA) and the University of Groningen as described previously [21].

Additionally, we assessed whether $\mathrm{CMH}$ was associated with mRNA expression of candidate genes in bronchial biopsies from 77 COPD participants in the Groningen and Leiden Universities Study of Corticosteroids in Obstructive Lung Disease study (GLUCOLD) [22, 23].

Details of the methods are given in the online supplementary material.

\section{Statistical analysis}

General characteristics of CMH cases and controls were compared using Student's t- and Mann-Whitney U-tests for continuous variables as appropriate, and using Chi-squared tests for dichotomous variables with SPSS 20.0 (IBM, Armonk, NY, USA). Quality control of genotyping, regression and meta-analyses were performed with PLINK 1.07 [24]. Quality control was performed in cases and controls according to the following exclusion criteria: SNPs with call rate $<95 \%$; minor allele frequency $<0.05$; proportion of individuals for which no genotype was called (mind) $<0.95$; and Hardy-Weinberg equilibrium $\mathrm{p}<0.0001$. Ethnic outliers, duplicates and relatives were removed (based on the top two components from multidimensional scaling).

Logistic regression analysis under an additive genetic model with adjustment for centre and smoking (ex/ current) was used to identify SNPs associated with CMH in NELSON participants in two separate analyses. SNPs were included for replication if there was any nominally significant association between $\mathrm{CMH}$ and a SNP $\left(\mathrm{p}<2.0 \times 10^{-4}\right)$, and analysed using additional adjustment for sex as the replication cohorts also included females.

\section{Results}

\section{Populations}

After quality control, out of 3005 NELSON participants, 2799 remained. Females were excluded as only 48 were present after quality control. 2194 NELSON males with complete information on CMH, spirometry and smoking history were analysed, including 849 with and 1345 without COPD. The prevalence of CMH in individuals with COPD was 39.8\% $(n=338)$ and in individuals without COPD 25.4\% ( $n=342)$. Demographic and clinical characteristics of NELSON participants with COPD and of the four COPD replication cohorts are presented in table 1 [17-20].

Demographic and clinical characteristics of NELSON participants without COPD and the replication cohort LifeLines are presented in table 2.

In all cohorts, irrespective of COPD status, individuals with $\mathrm{CMH}$ had significantly lower $\mathrm{FEV}_{1} \%$ predicted and were significantly more often current smokers than individuals without $\mathrm{CMH}$.

\section{Genome-wide analyses in NELSON participants with COPD}

After quality control, out of 620901 SNPs, 522636 remained for GWA analysis in 849 individuals with COPD, 338 with and 511 without CMH. The quantile-quantile (QQ)-plot showed no indication of 
TABLE 1 Characteristics of individuals with and without chronic mucus hypersecretion (CMH), in NELSON participants with chronic obstructive pulmonary disease (COPD) and in replication COPD cohorts

\begin{tabular}{|c|c|c|c|c|c|c|c|c|c|c|c|c|c|c|c|}
\hline & \multicolumn{3}{|c|}{ NELSON-COPD } & \multicolumn{12}{|c|}{ Replication cohort } \\
\hline & \multirow{2}{*}{$\mathrm{CMH}$} & \multirow{2}{*}{ No $\mathrm{CMH}$} & \multirow{2}{*}{ p-value } & \multicolumn{3}{|c|}{ GenKOLS } & \multicolumn{3}{|c|}{ COPDGene } & \multicolumn{3}{|c|}{ ECLIPSE } & \multicolumn{3}{|c|}{ MESA } \\
\hline & & & & $\mathrm{CMH}$ & No CMH & p-value & CMH & No CMH & p-value & CMH & No $\mathrm{CMH}$ & p-value & $\mathrm{CMH}$ & No CMH & p-value \\
\hline Subjects n (\%) & $\begin{array}{c}338 \\
(39.9)\end{array}$ & $\begin{array}{c}511 \\
(60.1)\end{array}$ & & $\begin{array}{c}487 \\
(57.1)\end{array}$ & $\begin{array}{c}364 \\
(42.7)\end{array}$ & & $\begin{array}{c}182 \\
(36.6)\end{array}$ & $\begin{array}{c}315 \\
(63.4)\end{array}$ & & $\begin{array}{c}643 \\
(38.1)\end{array}$ & $\begin{array}{c}1045 \\
(61.9)\end{array}$ & & $50(21.4)$ & $184(78.6)$ & \\
\hline Age years & $61.5 \pm 5.9$ & $61.2 \pm 5.4$ & 0.44 & $65.8 \pm 10.0$ & $65.2 \pm 10.0$ & 0.36 & $63.9 \pm 7.8$ & $65.2 \pm 8.3$ & 0.09 & $62.9 \pm 7.6$ & $64.1 \pm 6.8$ & 0.37 & $64.8 \pm 9.4$ & $65.6 \pm 9.1$ & 0.61 \\
\hline Females $\%$ & 0 & 0 & & 0 & 0 & & 39.0 & 57.1 & 0.001 & 24.7 & 38.5 & $<0.001$ & 58.0 & 64.7 & 0.39 \\
\hline Smoking pack-years & $\begin{array}{c}38.7 \\
(20-140)\end{array}$ & $\begin{array}{c}38.7 \\
(20-119)\end{array}$ & 0.044 & $\begin{array}{c}33.2 \\
(5-119)\end{array}$ & $\begin{array}{c}31.2 \\
(5-130)\end{array}$ & 0.16 & $\begin{array}{c}47.8 \\
(11-238)\end{array}$ & $\begin{array}{c}47.6 \\
(10-146)\end{array}$ & 0.16 & $\begin{array}{c}45.0 \\
(6-220)\end{array}$ & $\begin{array}{c}45.0 \\
(10-205)\end{array}$ & 0.10 & $\begin{array}{c}47.0 \\
(6-135)\end{array}$ & $\begin{array}{c}40.6 \\
(5-167)\end{array}$ & 0.19 \\
\hline Current smokers \% & 74.8 & 50.2 & $<0.001$ & 53.5 & 39.7 & $<0.001$ & 42.9 & 23.5 & $<0.001$ & 45.1 & 27.0 & $<0.001$ & 38.0 & 12.5 & $<0.001$ \\
\hline FEV $1 \%$ predicted & $81.8 \pm 19.8$ & $86.3 \pm 7.1$ & $<0.001$ & $48.2 \pm 17.5$ & $54.0 \pm 16.8$ & $<0.001$ & $46.5 \pm 18.1$ & $49.9 \pm 18.5$ & 0.044 & $46.7 \pm 15.4$ & $48.2 \pm 15.7$ & $<0.001$ & $67.5 \pm 18.6$ & $75.4 \pm 17.4$ & 0.005 \\
\hline $\mathrm{FEV}_{1} / \mathrm{FVC} \%$ & $60.1 \pm 8.6$ & $62.5 \pm 7.1$ & $<0.001$ & $49.7 \pm 13.4$ & $53.5 \pm 12.2$ & $<0.001$ & $45.5 \pm 11.9$ & $48.6 \pm 13.8$ & 0.007 & $44.3 \pm 11.8$ & $49.7 \pm 13.3$ & $<0.001$ & $59.4 \pm 10.5$ & $62.6 \pm 7.2$ & 0.014 \\
\hline
\end{tabular}

Data are presented as mean \pm SD or median (range), unless otherwise stated. FEV ${ }_{1}$ : forced expiratory volume in 1 s; FVC: forced vital capacity. 
TABLE 2 Characteristics of individuals with and without chronic mucus hypersecretion (CMH) in NELSON subjects without chronic obstructive pulmonary disease (COPD) and in the LifeLines cohort

\begin{tabular}{|c|c|c|c|c|c|c|}
\hline & \multicolumn{3}{|c|}{ NELSON-non-COPD } & \multicolumn{3}{|c|}{ LifeLines } \\
\hline Subjects n (\%) & 342 (25.4) & $1006(74.6)$ & & 130 (5.3) & 2313 (94.7) & \\
\hline Age years & $59.6 \pm 5.3$ & $59.8 \pm 5.3$ & 0.61 & $47.2 \pm 10.7$ & $47.4 \pm 9.7$ & 0.82 \\
\hline Females $\%$ & 0 & 0 & & 46.2 & 53.4 & 0.11 \\
\hline Smoking pack-years & $38.0(22-140)$ & $34.2(20-133)$ & 0.029 & $15.5(5-84)$ & $13.0(5-75)$ & $<0.001$ \\
\hline Current smokers \% & 70.8 & 45.2 & $<0.001$ & 60.0 & 43.1 & $<0.001$ \\
\hline
\end{tabular}

Data are presented as mean \pm SD or median (range), unless otherwise stated. $\mathrm{FEV}_{1}$ : forced expiratory volume in 1 s; FVC: forced vital capacity.

population stratification $(\lambda=1.002)$. The p-values of the GWA study are presented in the Manhattan plot (fig. 1). A total of 78 SNPs were associated with $\mathrm{CMH}$ at a $\mathrm{p}<2 \times 10^{-4}$ (table 3). SNP rs626326, located in an intron in the StAR-related lipid transfer domain containing 13 gene (STARD13) on chromosome 13q13.1, showed the strongest association with $\mathrm{CMH}\left(\mathrm{p}=3.99 \times 10^{-6}\right.$, OR 1.632).

When performing replication in males only, i.e. the same sex as in the identification cohort, results were comparable with all SNP effects in the same direction, but with lower significance due to the deletion of 714 females ( $23 \%$ of the population) and, hence, lower power.

\section{Replication of top SNPs in four COPD cohorts}

table 3 shows the results of the 78 SNPs that were analysed in 3106 individuals with COPD, including 1198 with and 1908 without CMH, participating in four different COPD cohorts. Meta-analyses of these 78 SNPs across the replication cohorts showed borderline association to six SNPs with CMH and a similar direction of effect (combined p-values ranging from $1.02 \times 10^{-2}$ to $9.49 \times 10^{-2}$ ).

The strongest association in the meta-analysis, across identification and replication cohorts, was observed for rs10461985 on chromosome 5p13.2, showing effects in the same direction in NELSON-COPD and the replication cohorts $\left(\mathrm{p}=5.43 \times 10^{-5}\right.$, OR 0.714$)$ (table 3), except for COPDGene, which showed no effect. SNP rs10461985 is located in an intron in the glial cell line-derived neurotrophic factor antisense RNA 1 gene (GDNF-AS1).

\section{Functional relevance of rs10461985 and GDNF}

The Affymetrix (Santa Clara, CA, USA) chip used to investigate mRNA expression in airway wall biopsies of COPD patients did not have probe set for GDNF-AS1. As the role of GDNF-AS1 as an antisense RNA is to prevent translation of GDNF, we assessed the association of the mRNA expression of this gene and
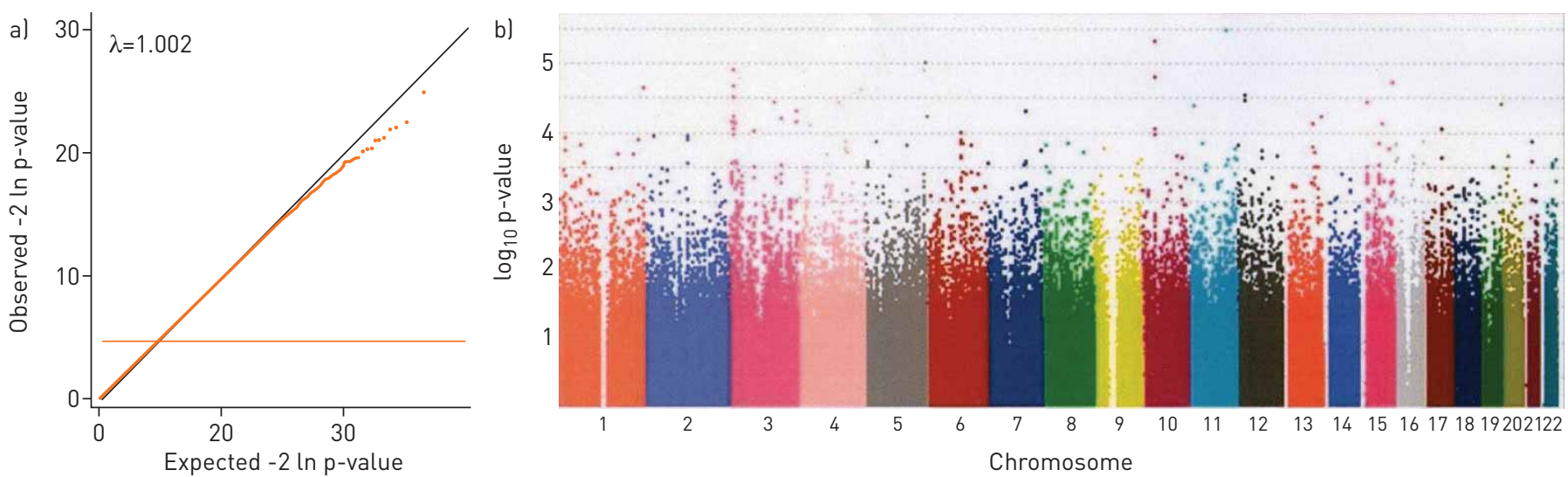

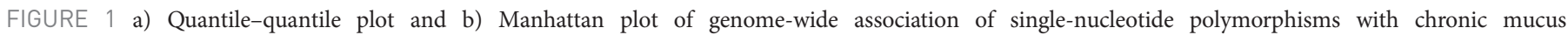
hypersecretion in NELSON participants with chronic obstructive pulmonary disease. 
TABLE 3 Association of single-nucleotide polymorphisms (SNPs) with chronic mucus hypersecretion in identification analysis (NELSON subjects with chronic obstructive pulmonary disease (COPDI) and in replication cohorts, and subsequent meta-analysis across identification and replication cohorts

CHR SNP

NELSON-COPD

\begin{tabular}{|c|c|c|c|c|c|c|c|c|c|c|c|c|}
\hline & & \multirow[t]{2}{*}{ Rank } & \multirow[t]{2}{*}{ p-value } & \multirow[t]{2}{*}{ OR } & \multicolumn{2}{|c|}{ GenKOLS } & \multicolumn{2}{|c|}{ COPDGene } & \multicolumn{2}{|c|}{ ECLIPSE } & \multicolumn{2}{|c|}{ MESA } \\
\hline & & & & & p-value & OR & $p$-value & OR & $p$-value & OR & $p$-value & OR \\
\hline 1 & rs2810587 & 33 & $9.90 \times 10^{-5}$ & 1.59 & $3.99 \times 10^{-1}$ & 1.10 & $3.10 \times 10^{-1}$ & 0.85 & $2.30 \times 10^{-1}$ & 0.90 & $6.49 \times 10^{-2}$ & 0.57 \\
\hline 1 & rs17518769 & 28 & $8.94 \times 10^{-5}$ & 2.03 & $1.49 \times 10^{-1}$ & 0.73 & 1.00 & 1.00 & $3.00 \times 10^{-1}$ & 1.15 & $8.11 \times 10^{-2}$ & 0.55 \\
\hline 1 & rs10753077 & 3 & $1.65 \times 10^{-5}$ & 1.79 & $4.95 \times 10^{-1}$ & 1.10 & $8.20 \times 10^{-1}$ & 1.05 & $6.70 \times 10^{-1}$ & 1.04 & $7.04 \times 10^{-1}$ & 1.15 \\
\hline 1 & rs12410049 & 49 & $1.38 \times 10^{-4}$ & 1.79 & $7.96 \times 10^{-1}$ & 1.04 & $4.20 \times 10^{-1}$ & 0.84 & $2.90 \times 10^{-1}$ & 0.88 & $9.02 \times 10^{-1}$ & 0.96 \\
\hline 1 & rs2001475 & 50 & $1.38 \times 10^{-4}$ & 1.79 & $7.96 \times 10^{-1}$ & 1.04 & $4.20 \times 10^{-1}$ & 0.84 & $2.90 \times 10^{-1}$ & 0.88 & $9.28 \times 10^{-1}$ & 0.97 \\
\hline 1 & rs3123695 & 36 & $1.08 \times 10^{-4}$ & 1.85 & $2.12 \times 10^{-1}$ & 0.78 & $7.40 \times 10^{-1}$ & 0.92 & $3.90 \times 10^{-1}$ & 0.90 & $6.49 \times 10^{-1}$ & 0.83 \\
\hline 2 & rs4671197 & 63 & $1.67 \times 10^{-4}$ & 1.50 & $6.85 \times 10^{-1}$ & 0.96 & $3.90 \times 10^{-1}$ & 1.15 & $3.90 \times 10^{-1}$ & 1.07 & $5.82 \times 10^{-1}$ & 0.86 \\
\hline 2 & rs216626 & 25 & $7.95 \times 10^{-5}$ & 1.89 & $2.44 \times 10^{-1}$ & 1.22 & $8.80 \times 10^{-1}$ & 1.03 & $2.50 \times 10^{-1}$ & 1.14 & $1.93 \times 10^{-1}$ & 0.67 \\
\hline 2 & rs216640 & 59 & $1.55 \times 10^{-4}$ & 1.86 & $2.55 \times 10^{-1}$ & 1.21 & $8.40 \times 10^{-1}$ & 1.04 & $2.70 \times 10^{-1}$ & 1.13 & $1.84 \times 10^{-1}$ & 0.67 \\
\hline 2 & rs3821072 & 20 & $6.69 \times 10-5$ & 1.93 & $2.00 \times 10^{-1}$ & 1.25 & $7.90 \times 10^{-1}$ & 1.06 & $3.50 \times 10^{-1}$ & 1.11 & $1.89 \times 10^{-1}$ & 0.67 \\
\hline 2 & rs6760631 & 68 & $1.78 \times 10^{-4}$ & 0.60 & $4.55 \times 10^{-1}$ & 0.91 & $5.00 \times 10^{-2}$ & 1.35 & $5.20 \times 10^{-1}$ & 1.06 & $4.37 \times 10^{-2}$ & 0.61 \\
\hline 3 & rs6442701 & 70 & $1.82 \times 10^{-4}$ & 0.66 & $7.29 \times 10^{-1}$ & 0.96 & $3.90 \times 10^{-1}$ & 0.88 & $9.50 \times 10^{-1}$ & 1.00 & $1.57 \times 10^{-1}$ & 1.45 \\
\hline 3 & rs6799163 & 73 & $1.90 \times 10^{-4}$ & 0.66 & $7.11 \times 10^{-1}$ & 0.96 & $4.70 \times 10^{-1}$ & 0.90 & $9.30 \times 10^{-1}$ & 0.99 & & \\
\hline 3 & rs492476 & 67 & $1.76 \times 10^{-4}$ & 0.64 & $1.14 \times 10^{-1}$ & 1.20 & $1.10 \times 10^{-1}$ & 1.28 & $7.90 \times 10^{-1}$ & 0.98 & $4.64 \times 10^{-1}$ & 1.24 \\
\hline 3 & rs4420851 & 69 & $1.80 \times 10^{-4}$ & 0.65 & $1.20 \times 10^{-1}$ & 1.19 & $1.30 \times 10^{-1}$ & 1.26 & $6.70 \times 10^{-1}$ & 0.96 & $4.79 \times 10^{-1}$ & 1.23 \\
\hline 3 & rs547906 & 39 & $1.13 \times 10^{-4}$ & 1.54 & $9.05 \times 10^{-1}$ & 0.99 & $7.00 \times 10^{-2}$ & 1.29 & $2.10 \times 10^{-1}$ & 0.90 & $9.57 \times 10^{-1}$ & 0.99 \\
\hline 3 & rs12632517 & 29 & $9.02 \times 10^{-5}$ & 1.56 & $9.23 \times 10^{-1}$ & 1.01 & $1.00 \times 10^{-1}$ & 1.27 & $5.00 \times 10^{-2}$ & 0.85 & $9.28 \times 10^{-1}$ & 0.98 \\
\hline 3 & rs4515036 & 40 & $1.16 \times 10^{-4}$ & 1.55 & $9.76 \times 10^{-1}$ & 1.00 & $1.00 \times 10^{-1}$ & 1.27 & $4.00 \times 10^{-2}$ & 0.85 & $9.28 \times 10^{-1}$ & 0.98 \\
\hline 3 & rs9826025 & 30 & $9.30 \times 10^{-5}$ & 1.56 & $8.16 \times 10^{-1}$ & 0.97 & $1.00 \times 10^{-1}$ & 1.27 & $4.00 \times 10^{-2}$ & 0.85 & $9.96 \times 10^{-1}$ & 1.00 \\
\hline 3 & rs3856798 & 66 & $1.74 \times 10^{-4}$ & 0.55 & $1.93 \times 10^{-1}$ & 1.21 & $5.50 \times 10^{-1}$ & 1.13 & $7.70 \times 10^{-1}$ & 1.03 & $2.33 \times 10^{-2}$ & 2.63 \\
\hline 3 & rs2447616 & 47 & $1.34 \times 10^{-4}$ & 0.54 & $2.02 \times 10^{-1}$ & 1.21 & $5.10 \times 10^{-1}$ & 1.14 & $7.60 \times 10^{-1}$ & 1.03 & $3.48 \times 10^{-2}$ & 2.52 \\
\hline 3 & rs9831604 & 55 & $1.47 \times 10^{-4}$ & 0.55 & $1.73 \times 10^{-1}$ & 1.22 & $5.10 \times 10^{-1}$ & 1.14 & $8.40 \times 10^{-1}$ & 1.02 & $2.30 \times 10^{-2}$ & 2.62 \\
\hline 3 & rs339668 & 34 & $1.02 \times 10^{-4}$ & 1.51 & $1.61 \times 10^{-1}$ & 1.15 & $2.00 \times 10^{-2}$ & 0.71 & $8.20 \times 10^{-1}$ & 1.02 & $4.08 \times 10^{-1}$ & 0.81 \\
\hline 3 & rs12485872 & 27 & $8.24 \times 10-5$ & 1.85 & $2.15 \times 10^{-1}$ & 0.84 & $6.70 \times 10^{-1}$ & 1.09 & $9.00 \times 10^{-1}$ & 1.01 & $5.27 \times 10^{-1}$ & 1.30 \\
\hline 4 & rs4306981 & 12 & $4.40 \times 10^{-5}$ & 1.57 & $4.84 \times 10^{-2}$ & 1.25 & $6.70 \times 10^{-1}$ & 0.94 & $8.90 \times 10^{-1}$ & 0.99 & $1.32 \times 10^{-1}$ & 1.52 \\
\hline 5 & rs7732527 & 43 & $1.25 \times 10^{-4}$ & 1.50 & $4.38 \times 10^{-1}$ & 1.08 & $8.00 \times 10^{-1}$ & 1.03 & $9.00 \times 10^{-1}$ & 1.01 & $7.12 \times 10^{-1}$ & 0.92 \\
\hline 5 & rs4867387 & 23 & $6.82 \times 10^{-5}$ & 1.73 & $4.28 \times 10^{-1}$ & 1.12 & $7.10 \times 10^{-1}$ & 0.92 & $6.50 \times 10^{-1}$ & 1.05 & $4.80 \times 10^{-1}$ & 1.27 \\
\hline 5 & rs11111 & 21 & $6.70 \times 10^{-5}$ & 0.56 & $7.72 \times 10^{-1}$ & 1.04 & $1.60 \times 10^{-1}$ & 0.76 & $2.40 \times 10^{-1}$ & 0.89 & $6.12 \times 10^{-1}$ & 0.84 \\
\hline 5 & rs10461985 & 71 & $1.82 \times 10^{-4}$ & 0.52 & $1.87 \times 10^{-1}$ & 0.78 & $9.80 \times 10^{-1}$ & 1.00 & $2.00 \times 10^{-2}$ & 0.74 & $3.70 \times 10^{-1}$ & 0.69 \\
\hline 5 & rs1501977 & 19 & $6.48 \times 10^{-5}$ & 0.62 & $1.94 \times 10^{-1}$ & 1.16 & $1.90 \times 10^{-1}$ & 0.81 & $6.00 \times 10^{-1}$ & 1.05 & $4.14 \times 10^{-1}$ & 0.78 \\
\hline 5 & rs1229729 & 52 & $1.42 \times 10^{-4}$ & 0.66 & $4.91 \times 10^{-1}$ & 1.07 & $2.50 \times 10^{-1}$ & 1.17 & $1.90 \times 10^{-1}$ & 1.11 & $9.62 \times 10^{-1}$ & 1.01 \\
\hline 5 & rs1229708 & 11 & $4.39 \times 10^{-5}$ & 1.54 & $8.06 \times 10^{-1}$ & 0.98 & $3.50 \times 10^{-1}$ & 0.88 & $7.60 \times 10^{-1}$ & 0.98 & $4.78 \times 10^{-1}$ & 1.19 \\
\hline 5 & rs7736228 & 74 & $1.91 \times 10^{-4}$ & 0.64 & $5.68 \times 10^{-1}$ & 0.94 & $1.70 \times 10^{-1}$ & 0.81 & $2.80 \times 10^{-1}$ & 0.91 & $7.86 \times 10^{-1}$ & 1.08 \\
\hline 5 & rs13178728 & 78 & $1.99 \times 10^{-4}$ & 1.91 & $8.49 \times 10^{-1}$ & 1.04 & $4.30 \times 10^{-1}$ & 1.22 & $9.70 \times 10^{-1}$ & 1.00 & $2.14 \times 10^{-1}$ & 1.80 \\
\hline 5 & rs13159558 & 56 & $1.49 \times 10^{-4}$ & 2.20 & $4.07 \times 10^{-1}$ & 1.18 & $7.50 \times 10^{-1}$ & 1.09 & $3.00 \times 10^{-1}$ & 0.87 & $4.90 \times 10^{-1}$ & 1.92 \\
\hline 6 & rs7751774 & 22 & $6.77 \times 10^{-5}$ & 0.52 & $2.06 \times 10^{-1}$ & 0.82 & $5.40 \times 10^{-1}$ & 0.88 & $7.50 \times 10^{-1}$ & 0.96 & $3.32 \times 10^{-1}$ & 0.72 \\
\hline 6 & rs1360811 & 14 & $5.80 \times 10^{-5}$ & 0.51 & $2.83 \times 10^{-1}$ & 0.84 & $4.10 \times 10^{-1}$ & 0.85 & $4.40 \times 10^{-1}$ & 0.92 & $4.82 \times 10^{-1}$ & 0.79 \\
\hline 6 & rs9503979 & 15 & $5.80 \times 10^{-5}$ & 0.51 & $2.88 \times 10^{-1}$ & 0.85 & $4.10 \times 10^{-1}$ & 0.84 & $4.10 \times 10^{-1}$ & 0.91 & $4.83 \times 10^{-1}$ & 0.79 \\
\hline 6 & rs6933317 & 31 & $9.44 \times 10^{-5}$ & 1.49 & $5.91 \times 10^{-1}$ & 0.95 & $6.90 \times 10^{-1}$ & 1.06 & $4.80 \times 10^{-1}$ & 1.06 & $8.54 \times 10^{-1}$ & 0.96 \\
\hline 6 & rs6940071 & 13 & $5.66 \times 10^{-5}$ & 1.52 & $9.38 \times 10^{-1}$ & 0.99 & $6.80 \times 10^{-1}$ & 1.06 & $1.30 \times 10^{-1}$ & 1.13 & $8.05 \times 10^{-1}$ & 0.94 \\
\hline 6 & rs12527298 & 64 & $1.69 \times 10^{-4}$ & 0.68 & $8.42 \times 10^{-1}$ & 0.98 & $7.70 \times 10^{-1}$ & 0.96 & $4.10 \times 10^{-1}$ & 0.94 & $9.54 \times 10^{-1}$ & 0.99 \\
\hline
\end{tabular}

Meta-analysis across identification

and replication cohorts

Rank p-value ${ }^{\#} \quad \mathrm{OR}^{\text {? }} \quad \mathbf{Q}$
Direction

of effect ${ }^{+}$

$\begin{array}{lccc}9.88 \times 10^{-1} & 1 & <0.001 & ++--- \\ 8.59 \times 10^{-1} & 1.04 & 0.001 & +-0+- \\ 5.44 \times 10^{-3} & 1.2 & 0.020 & +++0+ \\ 6.43 \times 10^{-1} & 1.07 & 0.004 & +0--- \\ 6.37 \times 10^{-1} & 1.08 & 0.004 & +0--0 \\ 8.84 \times 10^{-1} & 1.03 & 0.002 & +---- \\ 2.01 \times 10^{-2} & 1.13 & 0.030 & +0++- \\ 4.94 \times 10^{-3} & 1.23 & 0.016 & ++0+- \\ 8.06 \times 10^{-3} & 1.21 & 0.020 & ++0+- \\ 6.25 \times 10^{-3} & 1.22 & 0.013 & ++++- \\ 3.84 \times 10^{-1} & 0.88 & <0.001 & --++- \\ 5.92 \times 10^{-2} & 0.91 & 0.010 & -0-0+ \\ 2.44 \times 10^{-2} & 0.89 & 0.023 & -0-0 \times \\ 9.28 \times 10^{-1} & 1.01 & 0.001 & -++-+ \\ 9.95 \times 10^{-1} & 1 & 0.001 & -++0+ \\ 3.22 \times 10^{-1} & 1.12 & 0.002 & +0+-0 \\ 4.12 \times 10^{-1} & 1.11 & <0.001 & +0+-0 \\ 4.31 \times 10^{-1} & 1.11 & <0.001 & +0+-0 \\ 4.43 \times 10^{-1} & 1.11 & <0.001 & +0+-0 \\ 7.45 \times 10^{-1} & 1.09 & <0.001 & -++0+ \\ 8.37 \times 10^{-1} & 1.04 & <0.001 & -++0+ \\ 7.94 \times 10^{-1} & 1.05 & <0.001 & -++0+ \\ 7.58 \times 10^{-1} & 1.04 & 0.001 & ++-0- \\ 3.90 \times 10^{-1} & 1.21 & 0.003 & +-+00 \\ 4.12 \times 10^{-3} & 1.16 & 0.005 & ++-0+ \\ 2.46 \times 10^{-2} & 1.12 & 0.033 & ++00- \\ 7.70 \times 10^{-3} & 1.2 & 0.037 & ++-++ \\ 2.74 \times 10^{-3} & 0.82 & 0.033 & -0--- \\ 5.43 \times 10^{-5} & 0.71 & 0.228 & --0-- \\ 3.13 \times 10^{-1} & 0.88 & 0.001 & -+-+- \\ 8.80 \times 10^{-1} & 0.98 & 0.001 & -+++0 \\ 4.48 \times 10^{-1} & 1.08 & 0.003 & +0-0+ \\ 1.94 \times 10^{-3} & 0.85 & 0.100 & ----+ \\ 1.59 \times 10^{-2} & 1.23 & 0.037 & 00+0+ \\ 2.14 \times 10^{-3} & 1.48 & 0.101 & +++-+ \\ 2.23 \times 10^{-3} & 0.8 & 0.049 & ---0- \\ 1.50 \times 10^{-3} & 0.8 & 0.062 & ----- \\ 1.13 \times 10^{-3} & 0.79 & 0.070 & ----- \\ 3.09 \times 10^{-2} & 1.11 & 0.020 & +-++- \\ 3.46 \times 10^{-3} & 1.16 & 0.036 & +0++- \\ 1.34 \times 10^{-2} & 0.89 & 0.067 & -00-0 \\ & & & +0\end{array}$


Meta-analysis across identification

and replication cohorts

Direction

p-value $^{\#} \quad$ OR $^{\text {I }} \quad Q$

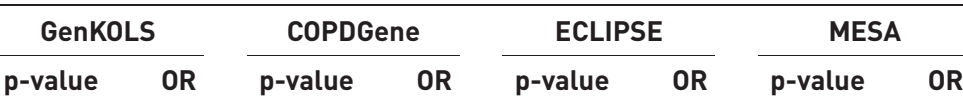

\begin{tabular}{|c|c|c|c|c|c|c|c|c|c|c|c|c|c|c|c|c|c|}
\hline 6 & rs12527846 & 53 & $1.42 \times 10^{-4}$ & 0.67 & $8.97 \times 10^{-1}$ & 0.99 & $7.70 \times 10^{-1}$ & 0.96 & $3.70 \times 10^{-1}$ & 0.93 & $8.92 \times 10^{-1}$ & 1.04 & 20 & $1.36 \times 10^{-2}$ & 0.86 & 0.037 & $-00-0$ \\
\hline 6 & rs12211633 & 76 & $1.95 \times 10^{-4}$ & 0.64 & $5.54 \times 10^{-1}$ & 0.94 & $7.20 \times 10^{-1}$ & 1.06 & $6.30 \times 10^{-1}$ & 1.04 & $2.18 \times 10^{-1}$ & 1.48 & 38 & $2.10 \times 10^{-1}$ & 0.94 & 0.006 & $--+0+$ \\
\hline 6 & rs2682185 & 51 & $1.38 \times 10^{-4}$ & 2.04 & $7.78 \times 10^{-1}$ & 1.05 & $9.90 \times 10^{-1}$ & 1.00 & $4.40 \times 10^{-1}$ & 1.11 & $4.50 \times 10^{-1}$ & 0.73 & 27 & $2.69 \times 10^{-2}$ & 1.21 & 0.028 & $++0+-$ \\
\hline 6 & rs164301 & 8 & $3.82 \times 10^{-5}$ & 0.64 & $9.34 \times 10^{-1}$ & 1.01 & $4.20 \times 10^{-1}$ & 1.12 & $8.70 \times 10^{-1}$ & 0.99 & $7.29 \times 10^{-1}$ & 1.09 & 51 & $5.14 \times 10^{-1}$ & 0.94 & 0.004 & $-0+0+$ \\
\hline 6 & rs9365242 & 5 & $2.55 \times 10^{-5}$ & 0.55 & $4.29 \times 10^{-1}$ & 0.91 & $5.20 \times 10^{-1}$ & 1.12 & $9.80 \times 10^{-1}$ & 1.00 & $9.84 \times 10^{-1}$ & 1.01 & 29 & $4.04 \times 10^{-2}$ & 0.88 & 0.006 & --+00 \\
\hline 6 & rs12055716 & 24 & $7.26 \times 10^{-5}$ & 0.59 & $5.95 \times 10^{-1}$ & 0.94 & $7.10 \times 10^{-1}$ & 1.06 & $5.40 \times 10^{-1}$ & 0.95 & $7.32 \times 10^{-1}$ & 1.11 & 23 & $1.97 \times 10^{-2}$ & 0.84 & 0.013 & --+-+ \\
\hline 6 & rs9295312 & 17 & $5.96 \times 10^{-5}$ & 1.84 & $7.19 \times 10^{-1}$ & 0.95 & $6.10 \times 10^{-1}$ & 0.91 & $2.90 \times 10^{-1}$ & 0.89 & $7.20 \times 10^{-1}$ & 1.13 & 54 & $5.64 \times 10^{-1}$ & 1.09 & 0.002 & +---+ \\
\hline 8 & rs4875186 & 42 & $1.23 \times 10^{-4}$ & 1.91 & $8.46 \times 10^{-1}$ & 0.97 & $6.80 \times 10^{-1}$ & 1.09 & $2.80 \times 10^{-1}$ & 0.87 & $8.81 \times 10^{-1}$ & 0.95 & 50 & $4.93 \times 10^{-1}$ & 1.12 & .004 & $+0+--$ \\
\hline 8 & rs7830870 & 16 & $5.81 \times 10^{-5}$ & 1.67 & $7.27 \times 10^{-1}$ & 1.04 & $1.00 \times 10^{-1}$ & 1.32 & $7.40 \times 10^{-1}$ & 1.03 & $6.98 \times 10^{-1}$ & 1.14 & 12 & $4.81 \times 10^{-3}$ & 1.18 & .024 & $+0+0+$ \\
\hline 8 & rs1864773 & 7 & $2.90 \times 10^{-5}$ & 1.88 & $9.14 \times 10^{-1}$ & 1.02 & $9.80 \times 10^{-1}$ & 0.99 & $8.80 \times 10^{-1}$ & 0.98 & $6.34 \times 10^{-1}$ & 1.18 & 31 & $4.62 \times 10^{-2}$ & 1.15 & .008 & $+000+$ \\
\hline 8 & rs7840848 & 37 & $1.10 \times 10^{-4}$ & 1.51 & $6.09 \times 10^{-1}$ & 1.05 & $5.60 \times 10^{-1}$ & 1.08 & $5.20 \times 10^{-1}$ & 0.95 & $4.29 \times 10^{-1}$ & 0.82 & 35 & $8.90 \times 10^{-2}$ & 1.09 & 0.008 & +++-- \\
\hline 8 & rs2289001 & 46 & $1.33 \times 10^{-4}$ & 1.53 & $8.58 \times 10^{-1}$ & 1.02 & $6.80 \times 10^{-1}$ & 1.07 & $3.30 \times 10^{-1}$ & 0.92 & $2.68 \times 10^{-1}$ & 1.38 & 37 & $1.27 \times 10^{-1}$ & 1.08 & 0.005 & $+0+-+$ \\
\hline 11 & rs6483640 & 75 & $1.93 \times 10^{-4}$ & 1.47 & $1.97 \times 10^{-1}$ & 1.14 & $5.80 \times 10^{-1}$ & 1.08 & $8.50 \times 10^{-1}$ & 1.02 & $7.15 \times 10^{-1}$ & 1.11 & 11 & $4.63 \times 10^{-3}$ & 1.15 & 0.088 & $+++0+$ \\
\hline 11 & rs2217032 & 54 & $1.43 \times 10^{-4}$ & 1.51 & $6.22 \times 10^{-1}$ & 1.05 & $3.00 \times 10^{-1}$ & 1.15 & $1.20 \times 10^{-1}$ & 1.13 & $9.30 \times 10^{-1}$ & 0.98 & 2 & $1.05 \times 10^{-3}$ & 1.18 & 0.119 & ++++- \\
\hline 11 & rs2292730 & 48 & $1.36 \times 10^{-4}$ & 0.67 & $8.59 \times 10^{-1}$ & 0.98 & $2.50 \times 10^{-1}$ & 0.85 & $4.60 \times 10^{-1}$ & 1.06 & $7.80 \times 10^{-2}$ & 1.61 & 56 & $5.89 \times 10^{-1}$ & 0.94 & 0.002 & $-0-++$ \\
\hline 11 & rs7935816 & 18 & $6.40 \times 10^{-5}$ & 0.63 & $1.64 \times 10^{-1}$ & 1.17 & $9.10 \times 10^{-1}$ & 0.98 & $1.40 \times 10^{-1}$ & 1.13 & $5.43 \times 10^{-1}$ & 0.84 & 59 & $6.36 \times 10^{-1}$ & 0.94 & $<0.001$ & $-+0+-$ \\
\hline 12 & rs7304675 & 77 & $1.95 \times 10^{-4}$ & 0.66 & $9.16 \times 10^{-1}$ & 0.99 & $8.90 \times 10^{-1}$ & 0.98 & $5.00 \times 10^{-1}$ & 1.05 & $1.13 \times 10^{-2}$ & 2.17 & 75 & $9.54 \times 10^{-1}$ & 0.99 & 0.001 & $-00++$ \\
\hline 12 & rs812512 & 35 & $1.07 \times 10^{-4}$ & 1.51 & $7.33 \times 10^{-1}$ & 0.97 & $7.90 \times 10^{-1}$ & 0.96 & $1.00 \times 10^{-2}$ & 0.81 & $3.94 \times 10^{-1}$ & 0.79 & 76 & $9.85 \times 10^{-1}$ & 1 & $<0.001$ & +---- \\
\hline 13 & rs495680 & 6 & $2.78 \times 10^{-5}$ & 0.63 & $4.08 \times 10^{-2}$ & 1.24 & $9.60 \times 10^{-1}$ & 1.01 & $6.00 \times 10^{-1}$ & 0.96 & $9.63 \times 10^{-1}$ & 1.01 & 58 & $6.30 \times 10^{-1}$ & 0.94 & $<0.001$ & -+000 \\
\hline 13 & rs626326 & 1 & $3.99 \times 10^{-6}$ & 1.63 & $9.16 \times 10^{-2}$ & 0.84 & $1.00 \times 10^{-1}$ & 0.79 & $8.60 \times 10^{-1}$ & 0.99 & $7.54 \times 10^{-1}$ & 0.93 & 74 & $9.42 \times 10^{-1}$ & 1.01 & $<0.001$ & +---- \\
\hline 13 & rs2858808 & 4 & $1.79 \times 10^{-5}$ & 0.60 & $5.85 \times 10^{-1}$ & 1.06 & $4.10 \times 10^{-1}$ & 0.88 & $7.30 \times 10^{-1}$ & 1.03 & $3.74 \times 10^{-1}$ & 1.25 & 49 & $4.82 \times 10^{-1}$ & 0.92 & 0.001 & $-+-0+$ \\
\hline 13 & rs523523 & 2 & $1.32 \times 10^{-5}$ & 0.64 & $3.31 \times 10^{-1}$ & 1.10 & $1.60 \times 10^{-1}$ & 1.22 & $8.70 \times 10^{-1}$ & 0.99 & $8.83 \times 10^{-1}$ & 1.04 & 64 & $7.49 \times 10^{-1}$ & 0.96 & $<0.001$ & -++00 \\
\hline 13 & rs2697092 & 57 & $1.49 \times 10^{-4}$ & 1.62 & $3.34 \times 10^{-1}$ & 1.12 & $3.30 \times 10^{-1}$ & 0.84 & $3.80 \times 10^{-1}$ & 1.09 & $9.15 \times 10^{-1}$ & 1.03 & 18 & $1.13 \times 10^{-2}$ & 1.16 & 0.029 & ++-+0 \\
\hline 15 & rs8041061 & 61 & $1.60 \times 10^{-4}$ & 1.47 & $8.00 \times 10^{-1}$ & 1.03 & $5.60 \times 10^{-1}$ & 1.08 & $9.40 \times 10^{-1}$ & 0.99 & $2.67 \times 10^{-1}$ & 0.76 & 34 & $6.83 \times 10^{-2}$ & 1.09 & 0.014 & $-0-0+$ \\
\hline 15 & rs809736 & 62 & $1.62 \times 10^{-4}$ & 0.64 & $9.12 \times 10^{-1}$ & 1.01 & $4.20 \times 10^{-1}$ & 0.87 & $8.10 \times 10^{-1}$ & 0.98 & $5.78 \times 10^{-1}$ & 1.17 & 30 & $4.35 \times 10^{-2}$ & 0.89 & 0.024 & $-0-0+$ \\
\hline 18 & rs8088174 & 72 & $1.87 \times 10^{-4}$ & 1.64 & $3.77 \times 10^{-2}$ & 0.76 & $8.30 \times 10^{-1}$ & 0.96 & $4.70 \times 10^{-1}$ & 0.93 & $8.24 \times 10^{-1}$ & 1.08 & 68 & $8.32 \times 10^{-1}$ & 1.03 & 0.001 & $+-0-+$ \\
\hline 20 & rs6085660 & 10 & $4.03 \times 10^{-5}$ & 1.55 & $2.42 \times 10^{-1}$ & 0.89 & $9.10 \times 10^{-1}$ & 0.98 & $1.10 \times 10^{-1}$ & 1.13 & $9.41 \times 10^{-1}$ & 0.98 & 42 & $3.69 \times 10^{-1}$ & 1.1 & 0.004 & $+-0+0$ \\
\hline 20 & rs1500545 & 60 & $1.59 \times 10^{-4}$ & 1.49 & $2.86 \times 10^{-1}$ & 0.90 & $9.90 \times 10^{-1}$ & 1.00 & $2.50 \times 10^{-1}$ & 1.09 & $6.86 \times 10^{-1}$ & 0.91 & 33 & $6.50 \times 10^{-2}$ & 1.1 & 0.010 & $+-0+-$ \\
\hline 20 & rs6055258 & 58 & $1.53 \times 10^{-4}$ & 0.67 & $2.57 \times 10^{-1}$ & 0.89 & $4.00 \times 10^{-2}$ & 1.34 & $2.70 \times 10^{-1}$ & 0.92 & $5.68 \times 10^{-1}$ & 1.16 & 66 & $7.87 \times 10^{-1}$ & 0.96 & 0.001 & --+-+ \\
\hline 20 & rs969111 & 45 & $1.27 \times 10^{-4}$ & 0.67 & $2.76 \times 10^{-1}$ & 0.90 & $4.00 \times 10^{-2}$ & 1.34 & $2.60 \times 10^{-1}$ & 0.92 & $4.90 \times 10^{-1}$ & 1.19 & 57 & $5.99 \times 10^{-1}$ & 0.94 & 0.002 & --+-+ \\
\hline 20 & rs1008096 & 44 & $1.26 \times 10^{-4}$ & 0.67 & $2.41 \times 10^{-1}$ & 0.89 & $4.00 \times 10^{-2}$ & 1.34 & $2.70 \times 10^{-1}$ & 0.92 & $4.85 \times 10^{-1}$ & 1.20 & 55 & $5.89 \times 10^{-1}$ & 0.94 & 0.002 & --+-+ \\
\hline 20 & rs6118681 & 38 & $1.12 \times 10^{-4}$ & 1.51 & $2.46 \times 10^{-1}$ & 0.89 & $4.20 \times 10^{-1}$ & 1.13 & $1.40 \times 10^{-1}$ & 0.89 & $6.16 \times 10^{-1}$ & 1.14 & 52 & $5.25 \times 10^{-1}$ & 1.08 & 0.001 & +-+-+ \\
\hline 20 & rs6141026 & 9 & $3.98 \times 10^{-5}$ & 1.69 & $5.32 \times 10^{-1}$ & 0.93 & $5.60 \times 10^{-1}$ & 1.11 & $4.30 \times 10^{-1}$ & 1.08 & $7.41 \times 10^{-1}$ & 1.10 & 22 & $1.73 \times 10^{-2}$ & 1.16 & 0.013 & +-+++ \\
\hline 20 & rs6081741 & 65 & $1.71 \times 10^{-4}$ & 0.63 & $9.73 \times 10^{-1}$ & 1.00 & $6.00 \times 10^{-1}$ & 1.08 & $7.80 \times 10^{-1}$ & 0.98 & $6.74 \times 10^{-1}$ & 1.14 & 36 & $1.05 \times 10^{-1}$ & 0.91 & 0.018 & $-0+0+$ \\
\hline 20 & rs6013773 & 41 & $1.18 \times 10^{-4}$ & 0.67 & $8.80 \times 10^{-1}$ & 1.02 & $1.90 \times 10^{-1}$ & 1.20 & $2.40 \times 10^{-1}$ & 1.09 & $6.22 \times 10^{-1}$ & 0.88 & 62 & $6.94 \times 10^{-1}$ & 0.96 & 0.002 & $-0++-$ \\
\hline 23 & rs5927035 & 32 & $9.52 \times 10$ & 1.78 & $1.76 \times 10^{-1}$ & 0.85 & & & $9.10 \times 1$ & 0.99 & & & 53 & $5.34 \times$ & 1.13 & $<0.001$ & $+-x 0 x$ \\
\hline 23 & rs2879751 & 26 & $8.10 \times 10^{-5}$ & 1.79 & & & & & $9.90 \times 10^{-1}$ & 1.00 & & & 41 & $3.24 \times 10^{-1}$ & 1.33 & 0.003 & $+x \times 0 x$ \\
\hline
\end{tabular}

An empty box indicates that the SNP was not analysed in the corresponding replication cohort. CHR: chromosome; $Q$ : $p$-value for heterogeneity. ${ }^{\#}$ : fixed $p$-value if $Q>0.005$ and random $\mathrm{p}$-value if $\mathrm{Q}<0.005$. \%: fixed odds ratio if $\mathrm{Q}>0.005$ and random odds ratio if $Q<0.005$. $^{+}$: in identification and replication cohorts is presented in the order NELSON-COPD, GenKOLS, COPDGene, ECLIPSE and MESA, where - indicates odds ratio $\leqslant 0.95,0$ indicates odds ratio $>0.95-\leqslant 1.05,+$ indicates odds ratio $>1.05$ and $x$ indicates "not applicable". 

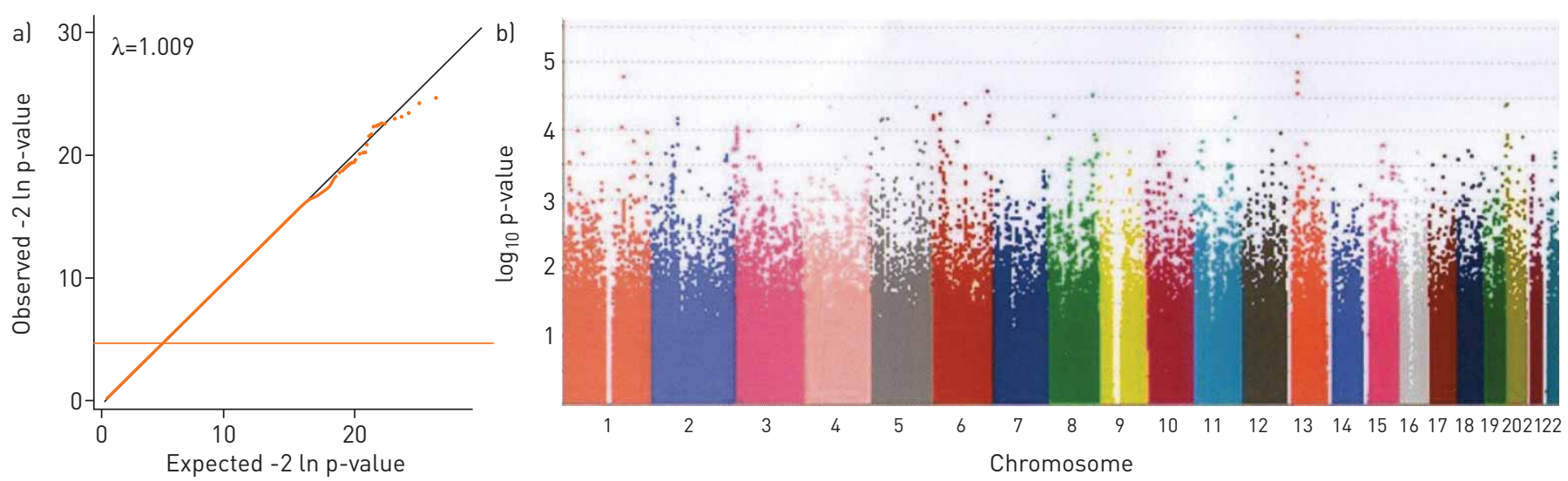

FIGURE 2 a) Quantile-quantile plot and b) Manhattan plot of genome-wide association of single-nucleotide polymorphisms with chronic mucus hypersecretion in NELSON participants without COPD.

CMH. GDNF mRNA expression was found to be significantly lower in bronchial biopsies of COPD patients with $\mathrm{CMH}$ than those without $\mathrm{CMH}(\mathrm{b}=-2.8, \mathrm{p}=0.007)$.

Genome-wide analyses in NELSON-non-COPD

The same 522636 SNPs were analysed in 1348 NELSON participants without COPD, 342 with and 1006 without CMH. The QQ-plot confirmed that there was no population stratification $(\lambda=1.009)$. The p-values of this GWA study are presented in the Manhattan plot (fig. 2). There were 79 SNPs associated with $\mathrm{CMH}$ with $\mathrm{p}<2.0 \times 10^{-4}$ (table 4 ).

Replication of top SNPs in the general population-based LifeLines cohort

Genotypes of 74 of the 79 SNPs with a $\mathrm{p}<2.0 \times 10^{-4}$ were available from the general population-based LifeLines cohort, including 130 individuals with $\mathrm{CMH}$ and 2313 without CMH. 10 SNPs showed some association with $\mathrm{CMH}$ in LifeLines $\left(\mathrm{p}<10^{-1}\right)$, and among these, seven SNPs had effects in the same direction in the NELSON participants without COPD and in LifeLines (table 4). In the meta-analysis across this NELSON population and LifeLines, four SNPs were associated with CMH with a $\mathrm{p}<10^{-5}$ : 1) rs3845529 on chromosome $1 \mathrm{q} 41\left(\mathrm{p}=3.25 \times 10^{-6}\right.$, OR 0.693$)$, located in an intron in the Usher syndrome $2 \mathrm{~A}$ gene $(U S H 2 A) ; 2)$ rs1690139 on chromosome $12 \mathrm{q}\left(\mathrm{p}=5.91 \times 10^{-6}\right.$, OR 1.673$)$, located in a gene desert between LOC100130336 and LOC100131830; 3) rs4863687 on chromosome 4q28 (p=7.57 $\times 10^{-6}$, OR 1.476), located in an intron in the mastermind-like 3 gene (MAML3); and 4) rs944899 on chromosome $13 \mathrm{q} 34\left(\mathrm{p}=8.40 \times 10^{-6}\right.$, OR 1.399), located near $(<25 \mathrm{~kb})$ the sex determining region Y-box 1 gene (SOX1).

Functional relevance of identified top SNPs associated with CMH in individuals without COPD

The rs3845529 genotypes showed no significant eQTL effect on USHA2 mRNA expression levels, nor did rs944899 genotypes on SOX1 mRNA expression levels, in lung tissue $\left(\mathrm{p} \approx 7 \times 10^{-1}\right)$. In contrast, a strong effect of rs4863687 genotypes (CC, $\mathrm{n}=622$; TC, $\mathrm{n}=408$; TT, $\mathrm{n}=66$ ) on MAML3 mRNA expression levels was shown; the $\mathrm{CMH}$-associated risk allele $\mathrm{T}$ was significantly associated with higher expression of MAML3 ( $\mathrm{p}=2.59 \times 10^{-12}$ ) (Affymetrix ID: 100146901-TGI-at; Ensemble ID: NM-018717) (fig. 3).

Gene expression profiles of genes close to rs1690139 were not present on the Affymetrix array for the eQTL analyses.

Overlap of top SNPs associated with CMH in COPD and non-COPD subjects

Comparison of top SNPs in the GWA studies in NELSON-COPD (5146 SNPs, $\mathrm{p}<10^{-2}$ ) and NELSON-non-COPD (5186 SNPs, $\mathrm{p}<10^{-2}$ ) showed 60 overlapping SNPs (table 5). When only SNPs with a p-value $<10^{-3}$ were considered, only one overlapping SNP was observed: rs4306981, located close to $(64 \mathrm{~kb})$ the progestin and adipoQ receptor family member III gene (PAQR3) on chromosome 4q21.21 ( $\mathrm{p}=4.40 \times 10^{-5}$ in individuals with COPD and $\mathrm{p}=5.73 \times 10^{-4}$ in those without COPD) with effects in the same direction in both analyses (OR 1.57 and 1.40, respectively). Follow up of this SNP in COPD cohorts did not confirm this association (meta-analysis across NELSON and replication cohorts $\mathrm{p}=4.12 \times 10^{-3}$ ). 


\begin{tabular}{|c|c|c|c|c|c|c|c|c|c|c|c|c|c|c|}
\hline \multirow[t]{2}{*}{ CHR } & \multirow[t]{2}{*}{ SNP } & \multirow[t]{2}{*}{ Position bp } & \multirow[t]{2}{*}{$\begin{array}{l}\text { Minor } \\
\text { allele }\end{array}$} & \multicolumn{4}{|c|}{ NELSON-non-COPD } & \multicolumn{2}{|c|}{ LifeLines } & \multicolumn{4}{|c|}{$\begin{array}{c}\text { Meta-analysis across } \\
\text { NELSON-non-COPD and LifeLines }\end{array}$} & \multirow[t]{2}{*}{ Closest gene(s) } \\
\hline & & & & MAF & Rank & $p$-value & $\mathrm{OR}$ & p-value & $\overline{O R}$ & Rank & p-value ${ }^{\#}$ & $\mathrm{OR}^{\pi}$ & $\mathbf{Q}$ & \\
\hline 1 & rs2817896 & 22988636 & G & 0.26 & 59 & $1.16 \times 10^{-4}$ & 1.47 & $1.09 \times 10^{-1}$ & 1.26 & 8 & $4.66 \times 10^{-5}$ & 1.40 & 0.362 & $E P H B 2^{\S}$ \\
\hline 1 & rs893961 & 22990760 & G & 0.25 & 66 & $1.81 \times 10^{-4}$ & 1.46 & $8.86 \times 10^{-2}$ & 1.28 & 9 & $5.30 \times 10^{-5}$ & 1.39 & 0.445 & $E P H B 2^{\S}$ \\
\hline 1 & rs11208807 & 66407509 & $A$ & 0.31 & 57 & $1.50 \times 10^{-4}$ & 1.43 & $2.55 \times 10^{-1}$ & 1.17 & 23 & $1.65 \times 10^{-4}$ & 1.34 & 0.228 & $P D E 4 B^{\S}$ \\
\hline 1 & rs2208370 & 170221954 & $A$ & 0.39 & 53 & $1.98 \times 10^{-4}$ & 1.42 & $7.22 \times 10^{-1}$ & 1.07 & 35 & $5.51 \times 10^{-4}$ & 1.33 & 0.154 & $D N M 3^{\S}$ \\
\hline 1 & rs3845529 & 214203243 & C & 0.42 & 73 & $1.96 \times 10^{-4}$ & 0.7 & $4.98 \times 10^{-3}$ & 0.67 & 1 & $3.25 \times 10^{-6}$ & 0.69 & 0.780 & USH $2 A^{\S}$ \\
\hline 1 & rs629199 & 232830726 & $A$ & 0.19 & 65 & $1.24 \times 10^{-4}$ & 1.54 & $3.64 \times 10^{-1}$ & 1.25 & 17 & $1.10 \times 10^{-4}$ & 1.48 & 0.445 & IRF2BP2, PP2672 \\
\hline 1 & rs12028329 & 245477414 & G & 0.25 & 46 & $2.20 \times 10^{-5}$ & 1.55 & $6.74 \times 10^{-1}$ & 1.07 & 21 & $1.47 \times 10^{-4}$ & 1.39 & 0.052 & LOC441931, VN1R5 \\
\hline 2 & rs1476151 & 125744258 & G & 0.46 & 19 & $1.08 \times 10^{-4}$ & 1.43 & $5.37 \times 10^{-1}$ & 0.91 & 62 & $2.98 \times 10^{-3}$ & 1.26 & 0.010 & CNTP5, LOC150554 \\
\hline 2 & rs13028050 & 125844903 & $A$ & 0.42 & 29 & $1.25 \times 10^{-4}$ & 0.7 & $7.36 \times 10^{-1}$ & 1.05 & 61 & $2.71 \times 10^{-3}$ & 0.79 & 0.016 & CNTP5, LOC150554 \\
\hline 3 & rs17776719 & 11615481 & G & 0.13 & 42 & $6.72 \times 10^{-5}$ & 1.64 & $5.58 \times 10^{-1}$ & 0.84 & 34 & $5.49 \times 10^{-4}$ & 1.49 & 0.038 & VGLL4§ \\
\hline 3 & rs2956507 & 13682301 & $A$ & 0.35 & 21 & $6.61 \times 10^{-5}$ & 0.68 & $7.82 \times 10^{-1}$ & 1.04 & 56 & $2.06 \times 10^{-3}$ & 0.78 & 0.011 & FBLN2, WNT7A \\
\hline 3 & rs6792244 & 13692200 & A & 0.42 & 28 & $5.77 \times 10^{-5}$ & 0.68 & $6.74 \times 10^{-1}$ & 1.07 & 49 & $1.28 \times 10^{-3}$ & 0.77 & 0.014 & FBLN2, WNT7A \\
\hline 3 & rs6775581 & 13695098 & G & 0.42 & 16 & $1.22 \times 10^{-5}$ & 0.66 & $6.80 \times 10^{-1}$ & 1.07 & 30 & $4.24 \times 10^{-4}$ & 0.75 & 0.009 & FBLN2, WNT7A \\
\hline 3 & rs6781368 & 13701841 & G & 0.43 & 14 & $2.02 \times 10^{-5}$ & 0.67 & $8.42 \times 10^{-1}$ & 1.03 & 42 & $8.12 \times 10^{-4}$ & 0.77 & 0.008 & FBLN2, WNT7A \\
\hline 3 & rs6794344 & 13701889 & $A$ & 0.46 & 24 & $8.84 \times 10^{-5}$ & 0.7 & $7.82 \times 10^{-1}$ & 1.04 & 59 & $2.51 \times 10^{-3}$ & 0.80 & 0.012 & FBLN2, WNT7A \\
\hline 3 & rs6795216 & 13705683 & C & 0.46 & 41 & $1.06 \times 10^{-4}$ & 0.7 & $9.03 \times 10^{-1}$ & 1.02 & 47 & $1.13 \times 10^{-3}$ & 0.77 & 0.035 & FBLN2, WNT7A \\
\hline 3 & rs2974399 & 13740911 & $A$ & 0.45 & 30 & $2.89 \times 10^{-5}$ & 0.68 & $7.99 \times 10^{-1}$ & 1.04 & 33 & $5.38 \times 10^{-4}$ & 0.76 & 0.018 & FBLN2, WNT7A \\
\hline 3 & rs6768597 & 20394587 & G & 0.3 & 50 & $7.05 \times 10^{-5}$ & 0.66 & $3.17 \times 10^{-1}$ & 0.87 & 20 & $1.44 \times 10^{-4}$ & 0.73 & 0.125 & SGOL1, VENTXP7 \\
\hline 3 & rs9682418 & 72180217 & G & 0.27 & 70 & $9.15 \times 10^{-5}$ & 1.48 & $4.91 \times 10^{-2}$ & 1.32 & 5 & $1.52 \times 10^{-5}$ & 1.43 & 0.494 & PROK2, CCDC137P \\
\hline 3 & rs11714053 & 133332100 & $A$ & 0.17 & 37 & $3.49 \times 10^{-5}$ & 1.61 & $5.06 \times 10^{-1}$ & 0.84 & 28 & $3.74 \times 10^{-4}$ & 1.46 & 0.026 & CPNE4, LOC729674 \\
\hline 3 & rs1403428 & 149752754 & $A$ & 0.22 & 52 & $5.96 \times 10^{-5}$ & 1.55 & $3.27 \times 10^{-1}$ & 1.16 & 19 & $1.18 \times 10^{-4}$ & 1.41 & 0.133 & LOC344741, RPL38P1 \\
\hline 3 & rs9825199 & 196385873 & A & 0.06 & 17 & $4.83 \times 10^{-5}$ & 2.02 & $4.88 \times 10^{-1}$ & 0.81 & 50 & $1.38 \times 10^{-3}$ & 1.62 & 0.009 & C3orf $21^{\S}$ \\
\hline 3 & rs3796160 & 196387903 & A & 0.06 & 22 & $6.76 \times 10^{-5}$ & 2 & $5.17 \times 10^{-1}$ & 0.82 & 52 & $1.74 \times 10^{-3}$ & 1.60 & 0.011 & C 3 orf $21^{\S}$ \\
\hline 4 & rs17447715 & 80821889 & A & 0.19 & 58 & $1.94 \times 10^{-4}$ & 0.62 & $1.52 \times 10^{-1}$ & 0.78 & 18 & $1.16 \times 10^{-4}$ & 0.67 & 0.295 & OR7E94P, GDEP \\
\hline 4 & rs6858670 & 137477830 & G & 0.47 & 32 & $1.29 \times 10^{-4}$ & 1.42 & $9.08 \times 10^{-1}$ & 0.99 & 57 & $2.13 \times 10^{-3}$ & 1.26 & 0.022 & LOC100132574, LOC646316 \\
\hline 4 & rs7688325 & 137479502 & $A$ & 0.47 & 35 & $1.65 \times 10^{-4}$ & 1.41 & $8.99 \times 10^{-1}$ & 0.98 & 60 & $2.54 \times 10^{-3}$ & 1.25 & 0.024 & LOC100132574, LOC646316 \\
\hline 4 & rs4863687 & 140897731 & $A$ & 0.28 & 72 & $1.89 \times 10^{-4}$ & 1.45 & $1.22 \times 10^{-2}$ & 1.57 & 3 & $7.57 \times 10^{-6}$ & 1.48 & 0.688 & $M A M L 3^{\S}$ \\
\hline 4 & rs6552407 & 181166606 & $A$ & 0.25 & 1 & $2.38 \times 10^{-5}$ & 1.55 & $7.85 \times 10^{-2}$ & 0.76 & 73 & $8.04 \times 10^{-1}$ & 1.09 & 0.000 & LOC391719, hCG_2025798 \\
\hline 5 & rs1816237 & 33076569 & G & 0.11 & 49 & $1.27 \times 10^{-4}$ & 0.53 & $8.00 \times 10^{-1}$ & 0.93 & 32 & $5.09 \times 10^{-4}$ & 0.61 & 0.102 & LOC340113, LOC̄728553 \\
\hline 5 & rs4836527 & 122670280 & $A$ & 0.4 & 33 & $1.45 \times 10^{-4}$ & 1.41 & $5.38 \times 10^{-1}$ & 0.9 & 54 & $1.96 \times 10^{-3}$ & 1.28 & 0.022 & PRDM6, CEP120 \\
\hline 5 & rs13183447 & 172004970 & A & 0.39 & 4 & $9.28 \times 10^{-6}$ & 0.65 & $3.04 \times 10^{-1}$ & 1.17 & 70 & $6.13 \times 10^{-1}$ & 0.86 & 0.001 & SH3PXD2B, LOC100130394 \\
\hline 5 & rs262020 & 177896923 & A & 0.39 & 54 & $5.78 \times 10^{-5}$ & 0.68 & $8.99 \times 10^{-1}$ & 0.97 & 24 & $1.68 \times 10^{-4}$ & 0.71 & 0.154 & COL23A1§ \\
\hline 6 & rs7770889 & 96965174 & A & 0.37 & 60 & $9.92 \times 10^{-5}$ & 1.45 & $3.65 \times 10^{-1}$ & 1.19 & 13 & $9.81 \times 10^{-5}$ & 1.40 & 0.368 & FUT9, KIAA0776 \\
\hline 6 & rs9486181 & 96974853 & G & 0.36 & 63 & $1.30 \times 10^{-4}$ & 1.45 & $2.82 \times 10^{-1}$ & 1.22 & 14 & $1.03 \times 10^{-4}$ & 1.40 & 0.410 & FUT9, KIAA0776 \\
\hline 6 & rs4425602 & 97000627 & G & 0.36 & 61 & $1.30 \times 10^{-4}$ & 1.45 & $2.93 \times 10^{-1}$ & 1.21 & 16 & $1.08 \times 10^{-4}$ & 1.39 & 0.396 & FUT9, KIAA0776 \\
\hline 6 & rs3860243 & 97012024 & A & 0.36 & 62 & $1.21 \times 10^{-4}$ & 1.45 & $2.79 \times 10^{-1}$ & 1.22 & 12 & $9.32 \times 10^{-5}$ & 1.40 & 0.402 & FUT9, KIAA0776 \\
\hline 6 & rs12207471 & 97070503 & A & 0.36 & 47 & $1.30 \times 10^{-4}$ & 1.45 & $9.17 \times 10^{-1}$ & 1.02 & 43 & $8.20 \times 10^{-4}$ & 1.32 & 0.064 & FUT9, KIAA0776 \\
\hline 6 & rs9398148 & 97170276 & G & 0.34 & 64 & $1.39 \times 10^{-4}$ & 1.45 & $2.97 \times 10^{-1}$ & 1.23 & 15 & $1.05 \times 10^{-4}$ & 1.40 & 0.442 & $F H L 5^{\S}$ \\
\hline 6 & rs9375195 & 98669441 & G & 0.48 & 40 & $1.35 \times 10^{-4}$ & 1.42 & $9.58 \times 10^{-1}$ & 1.01 & 53 & $1.78 \times 10^{-3}$ & 1.26 & 0.029 & C6orf167, LOC100129158 \\
\hline 6 & rs2151522 & 127251786 & $A$ & 0.39 & 55 & $1.45 \times 10^{-4}$ & 1.43 & $2.21 \times 10^{-1}$ & 1.17 & 22 & $1.57 \times 10^{-4}$ & 1.33 & 0.196 & LOC442257, RSPO3 \\
\hline 7 & rs10499977 & 108947923 & $A$ & 0.33 & 31 & $4.81 \times 10^{-5}$ & 1.48 & $6.02 \times 10^{-1}$ & 0.91 & 41 & $7.41 \times 10^{-4}$ & 1.34 & 0.020 & LOC646614, LOC100128056 \\
\hline 7 & rs12538214 & 154969302 & $A$ & 0.25 & 48 & $1.75 \times 10^{-4}$ & 1.48 & $5.29 \times 10^{-1}$ & 1.1 & 40 & $6.48 \times 10^{-4}$ & 1.34 & 0.092 & EN2, CNPY1 \\
\hline
\end{tabular}




\begin{tabular}{|c|c|c|c|c|c|c|c|c|c|c|c|c|c|c|}
\hline \multirow[t]{2}{*}{ CHR } & \multirow[t]{2}{*}{ SNP } & \multirow[t]{2}{*}{ Position bp } & \multirow[t]{2}{*}{$\begin{array}{l}\text { Minor } \\
\text { allele }\end{array}$} & \multicolumn{4}{|c|}{ NELSON-non-COPD } & \multicolumn{2}{|c|}{ LifeLines } & \multicolumn{4}{|c|}{$\begin{array}{c}\text { Meta-analysis across } \\
\text { NELSON-non-COPD and LifeLines }\end{array}$} & \multirow[t]{2}{*}{ Closest gene(s) } \\
\hline & & & & MAF & Rank & p-value & OR & p-value & OR & Rank & p-value $\#$ & $O R^{\pi}$ & $\mathbf{Q}$ & \\
\hline 8 & rs7007974 & 8839477 & G & 0.1 & 56 & $1.48 \times 10^{-4}$ & 1.69 & $2.75 \times 10^{-1}$ & 1.24 & 25 & $1.82 \times 10^{-4}$ & 1.53 & 0.208 & MRPS18CP2, LOC645960 \\
\hline 8 & rs13265648 & 73208111 & $A$ & 0.49 & 2 & $1.38 \times 10^{-4}$ & 0.7 & $8.67 \times 10^{-2}$ & 1.25 & 72 & $7.98 \times 10^{-1}$ & 0.93 & 0.000 & TRPA1, LOC392232 \\
\hline 8 & rs16886291 & 115780612 & A & 0.12 & 44 & $1.90 \times 10^{-4}$ & 0.55 & $6.96 \times 10^{-1}$ & 0.92 & 51 & $1.46 \times 10^{-3}$ & 0.67 & 0.047 & hCG_1644355, TRPS1 \\
\hline 9 & rs10119913 & 29254328 & c & 0.3 & 3 & $1.61 \times 10^{-4}$ & 0.68 & $5.54 \times 10^{-2}$ & 1.5 & 74 & $9.74 \times 10^{-1}$ & 0.99 & 0.001 & LINGO2, LOC286239 \\
\hline 10 & rs10827563 & 36255556 & G & 0.48 & 38 & $1.04 \times 10^{-4}$ & 1.43 & $5.15 \times 10^{-1}$ & 0.88 & 48 & $1.14 \times 10^{-3}$ & 1.31 & 0.027 & LOC439954, PBEF2 \\
\hline 10 & rs2696310 & 36262016 & G & 0.44 & 7 & $1.55 \times 10^{-5}$ & 1.5 & $6.65 \times 10^{-1}$ & 0.95 & 68 & $4.27 \times 10^{-1}$ & 1.20 & 0.004 & LOC439954, PBEF2 \\
\hline 10 & rs2767073 & 36269018 & $A$ & 0.44 & 8 & $4.75 \times 10^{-6}$ & 1.54 & $5.86 \times 10^{-1}$ & 0.92 & 26 & $2.21 \times 10^{-4}$ & 1.35 & 0.006 & LOC439954, PBEF2 \\
\hline 10 & rs1571136 & 36270927 & G & 0.44 & 18 & $1.57 \times 10^{-5}$ & 1.5 & $6.14 \times 10^{-1}$ & 0.92 & 31 & $4.56 \times 10^{-4}$ & 1.33 & 0.010 & LOC439954, PBEF2 \\
\hline 10 & rs2804852 & 36277541 & $A$ & 0.42 & 39 & $8.39 \times 10^{-5}$ & 1.44 & $6.53 \times 10^{-1}$ & 0.92 & 45 & $1.01 \times 10^{-3}$ & 1.31 & 0.028 & LOC439954, PBEF2 \\
\hline 11 & rs2071461 & 11330536 & $G$ & 0.24 & 26 & $3.86 \times 10^{-5}$ & 1.52 & $3.12 \times 10^{-1}$ & 0.78 & 37 & $6.06 \times 10^{-4}$ & 1.38 & 0.013 & GALNTL4 $4^{\S}$ \\
\hline 11 & rs3903687 & 35288218 & $G$ & 0.37 & 10 & $1.40 \times 10^{-4}$ & 1.43 & $4.90 \times 10^{-1}$ & 0.91 & 67 & $6.03 \times 10^{-3}$ & 1.24 & 0.006 & SLC1A2 \\
\hline 11 & rs474158 & 105342254 & $A$ & 0.07 & 36 & $3.28 \times 10^{-6}$ & 2.17 & $7.05 \times 10^{-1}$ & 1.1 & 7 & $4.35 \times 10^{-5}$ & 1.76 & 0.024 & GRIA4§ \\
\hline 11 & rs2288403 & 129243199 & G & 0.17 & 71 & $1.63 \times 10^{-4}$ & 0.6 & $6.27 \times 10^{-2}$ & 0.69 & 6 & $3.00 \times 10^{-5}$ & 0.63 & 0.604 & $N F R K B^{\S}$ \\
\hline 12 & rs10459134 & 5750112 & $A$ & 0.18 & 13 & $1.47 \times 10^{-4}$ & 1.55 & $5.12 \times 10^{-1}$ & 0.89 & 65 & $5.21 \times 10^{-3}$ & 1.31 & 0.008 & TMEM16B ${ }^{\S}$ \\
\hline 12 & rs7959932 & 23931073 & G & 0.32 & 9 & $2.74 \times 10^{-5}$ & 1.49 & $2.08 \times 10^{-1}$ & 0.74 & 39 & $6.34 \times 10^{-4}$ & 1.35 & 0.006 & SOX5§ \\
\hline 12 & rs7308636 & 23942557 & $A$ & 0.31 & 15 & $3.27 \times 10^{-5}$ & 1.48 & $2.34 \times 10^{-1}$ & 0.75 & 38 & $6.25 \times 10^{-4}$ & 1.35 & 0.008 & $50 \times 5^{\S}$ \\
\hline 12 & rs1690139 & 74558944 & G & 0.11 & 74 & $1.76 \times 10^{-4}$ & 1.67 & $1.11 \times 10^{-2}$ & 1.69 & 2 & $5.91 \times 10^{-6}$ & 1.67 & 0.951 & LOC100130336, LOC100131830 \\
\hline 13 & rs9300394 & 86801456 & $A$ & 0.29 & 27 & $1.52 \times 10^{-4}$ & 0.67 & $6.11 \times 10^{-1}$ & 1.09 & 64 & $3.67 \times 10^{-3}$ & 0.77 & 0.013 & LOC100130117, hCG_1795283 \\
\hline 13 & rs4514531 & 86805556 & G & 0.29 & 23 & $7.12 \times 10^{-5}$ & 0.66 & $6.32 \times 10^{-1}$ & 1.08 & 55 & $1.99 \times 10^{-3}$ & 0.76 & 0.011 & LOC100130117, hCG_1795283 \\
\hline 13 & rs944899 & 111798962 & $A$ & 0.46 & 69 & $5.76 \times 10^{-5}$ & 1.46 & $4.05 \times 10^{-2}$ & 1.3 & 4 & $8.40 \times 10^{-6}$ & 1.40 & 0.476 & SOX1 \\
\hline 15 & rs12594495 & 20499445 & G & 0.26 & 6 & $3.44 \times 10^{-5}$ & 0.62 & $5.49 \times 10^{-1}$ & 1.09 & 69 & $4.71 \times 10^{-1}$ & 0.82 & 0.002 & CYFIP1§ \\
\hline 15 & rs8042800 & 57638092 & $A$ & 0.3 & 5 & $1.36 \times 10^{-4}$ & 0.67 & $2.60 \times 10^{-1}$ & 1.17 & 71 & $6.39 \times 10^{-1}$ & 0.88 & 0.001 & FAM81A, GCNT3 \\
\hline 15 & rs3784350 & 66429101 & A & 0.37 & 11 & $7.25 \times 10^{-5}$ & 0.68 & $6.38 \times 10^{-1}$ & 1.07 & 63 & $3.47 \times 10^{-3}$ & 0.79 & 0.006 & ITGA11§ \\
\hline 15 & rs1348533 & 84527598 & A & 0.2 & 12 & $1.67 \times 10^{-4}$ & 0.63 & $4.36 \times 10^{-1}$ & 1.17 & 66 & $5.73 \times 10^{-3}$ & 0.75 & 0.008 & $A G B L 1$ \\
\hline 15 & rs8043332 & 96890829 & A & 0.3 & 20 & $1.85 \times 10^{-5}$ & 1.51 & $3.68 \times 10^{-1}$ & 0.82 & 29 & $3.84 \times 10^{-4}$ & 1.36 & 0.011 & FAM169B, IGF1R \\
\hline 16 & rs1978316 & 6277315 & A & 0.19 & 67 & $1.44 \times 10^{-4}$ & 1.53 & $1.85 \times 10^{-1}$ & 1.29 & 11 & $7.70 \times 10^{-5}$ & 1.46 & 0.448 & $A 2 B P 1^{\S}$ \\
\hline 16 & rs1344471 & 6278829 & A & 0.19 & 68 & $1.36 \times 10^{-4}$ & 1.53 & $1.84 \times 10^{-1}$ & 1.29 & 10 & $7.31 \times 10^{-5}$ & 1.47 & 0.449 & $A 2 B P 1^{\S}$ \\
\hline 16 & rs12443545 & 82156133 & A & 0.19 & 45 & $1.31 \times 10^{-4}$ & 0.62 & $5.94 \times 10^{-1}$ & 1.18 & 44 & $8.58 \times 10^{-4}$ & 0.68 & 0.051 & $\mathrm{CDH} 13^{\S}$ \\
\hline 16 & rs12918351 & 82156354 & G & 0.2 & 43 & $1.30 \times 10^{-4}$ & 0.62 & $9.35 \times 10^{-1}$ & 0.98 & 46 & $1.12 \times 10^{-3}$ & 0.71 & 0.044 & $\mathrm{CDH} 13^{\S}$ \\
\hline 17 & rs1508960 & 49024530 & G & 0.3 & 25 & $8.74 \times 10^{-5}$ & 1.45 & $7.06 \times 10^{-1}$ & 0.95 & 58 & $2.36 \times 10^{-3}$ & 1.27 & 0.012 & LOC645163, LOC645173 \\
\hline 20 & rs6042209 & 1354212 & $A$ & 0.18 & 34 & $3.64 \times 10^{-5}$ & 1.59 & $9.79 \times 10^{-1}$ & 1 & 36 & $5.69 \times 10^{-4}$ & 1.38 & 0.023 & FKBP1A, NSFL1C \\
\hline 21 & rs2032257 & 26696741 & A & 0.39 & 51 & $1.30 \times 10^{-4}$ & 0.69 & $3.58 \times 10^{-1}$ & 0.88 & 27 & $2.78 \times 10^{-4}$ & 0.75 & 0.131 & $A P P, C Y Y R 1$ \\
\hline
\end{tabular}

CHR: chromosome; MAF: minor allele frequency. $Q$ : $p$-value for heterogeneity. " : fixed $p$-value if $Q>0.005$ and random $p$-value if $Q<0.005 ;{ }^{~ "}$ : fixed odds ratio if $Q>0.005$ and random odds ratio if $Q<0.005 ;{ }^{\S}$ : SNP present in intron. 


\section{Discussion}

In the current study, we performed two separate GWA studies on smoking-induced $\mathrm{CMH}$, one in individuals with COPD and another in individuals without COPD. We did not find genome-wide significance for $\mathrm{CMH}$ in either individuals with COPD or without COPD. However, we found suggestive evidence of an association of some genes with $\mathrm{CMH}$ and differential mRNA expression for some of these genes. Different genes were associated with $\mathrm{CMH}$ in smokers with and without COPD. We found one overlapping SNP associated with CMH in NELSON-COPD and NELSON-non-COPD with a p-value $<10^{-3}$, yet this was not replicated in the validation cohorts. Together, our data raise the possibility that the pathogenetic development of $\mathrm{CMH}$ is differentially regulated in individuals with and without COPD.

In the analysis of CMH performed in individuals with COPD, we found one SNP, rs10461985, in GDNF-AS1 that had a lower $p$-value in the replication cohorts than in the identification analysis $\left(\mathrm{p}=5.43 \times 10^{-5}\right.$ and $\mathrm{p}=1.82 \times 10^{-4}$, respectively), showing the same direction of effect in all cohorts except one separately. Unfortunately, we were not able to perform a relevant study to assess the expression of GDNF-AS1 in bronchial biopsies of COPD-patients with and without CMH, as GDNF-AS1 was not present on the Affymetrix chip used to investigate mRNA expression in COPD patients (GLUCOLD). Antisense RNAs are transcribed to prevent translation of a complementary mRNA by base pairing to it and blocking translation [25]. In this way, GDNF-AS1 prevents expression of GDNF. When assessing the effect of rs10461985 in GDNF-AS1 on GDNF expression, we found no significant effect. However, this is not relevant in this context, as the effect of rs 10461985 is post-transcription, i.e. translational. It remains to be established whether the lower GDNF expression in bronchial biopsies of COPD patients with $\mathrm{CMH}$ is due to changes in translation of GDNF caused by GDNF-AS1. This requires further study. GDNF is a neurotrophic factor that can induce plasticity in sensory neurons innervating the respiratory tract and is involved in lung development [26-28]. These data suggest that GDNF is a biologically plausible candidate gene for both COPD and CMH. However, the gene has not been identified in previous GWA studies of lung function or COPD, making it more likely that it is a gene related to $\mathrm{CMH}$ in those who have $\mathrm{COPD}$ or a gene that interacts with genes associated with COPD. We did not have sufficient power to investigate further the latter possibility.

The SNP rs4863687, which is located in the MAML3 gene on chromosome 4, a transcriptional co-activator for Notch signalling, was associated with $\mathrm{CMH}$ in individuals without COPD. It has been suggested that MAML3 interacts functionally with different transcription factors, including $\beta$-catenin and NF- KB, both of which are associated with lung inflammation [29]. We found a strong effect of rs 4863687 genotype on $M A M L 3$ mRNA expression levels; the risk allele $\mathrm{T}$ was significantly associated with higher expression of MAML3. These data suggest that MAML3 affects risk of $\mathrm{CMH}$ by influencing inflammation. Additionally, it was shown in mice that coordinated cooperation between Wnt and Notch signalling in intestinal epithelium is necessary for the maintenance of proliferative cells, and that disruption of the Notch signalling pathway induces goblet cell conversion of crypt proliferative cells [30]. It is conceivable that the role of the Notch signalling pathway is also important in the airway epithelium, and that MAML3 may play a role in goblet cell hyperplasia and consequently CMH.

rs944899 was associated with CMH in individuals without COPD. It is located close to the SOX1 gene that belongs to a family of transcription factors involved in many tissues and developmental processes. SOX proteins have unique functions in different cell types and different functions within the same cell type.

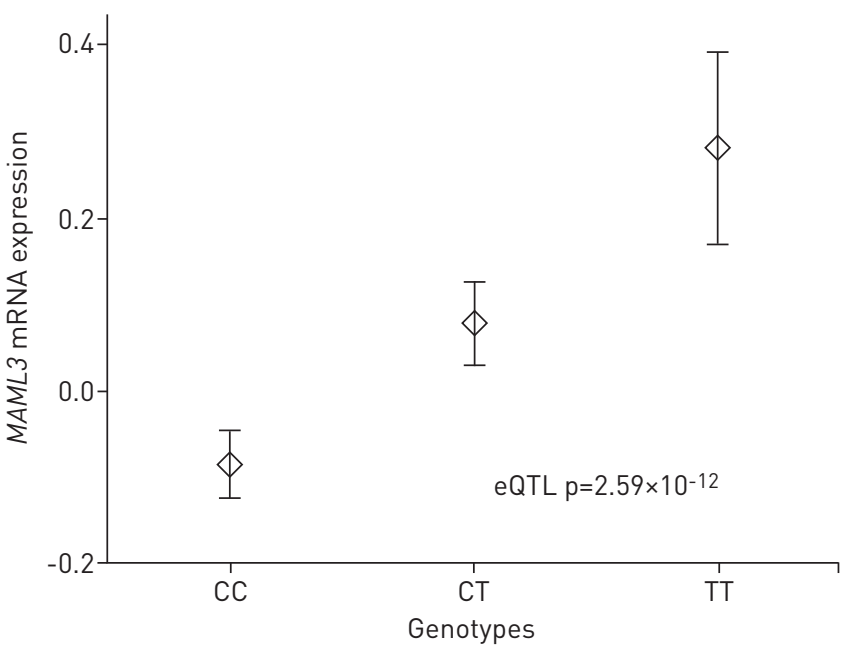

FIGURE 3 Lung gene expression levels of MAML3 according to genotype of the single-nucleotide polymorphism rs4868687 in 1095 individuals. CC, $\mathrm{n}=622 ; \mathrm{CT}, \mathrm{n}=408$; TT, $\mathrm{n}=65$. eQTL: expression quantitative trait locus. 
TABLE 5 Comparison of single-nucleotide polymorphisms (SNPs) associated with chronic mucus hypersecretion with a p-value $<10^{-2}$ in NELSON subjects with and without chronic obstructive pulmonary disease (COPD)

\begin{tabular}{|c|c|c|c|c|c|c|c|c|c|c|c|c|c|}
\hline \multirow[t]{2}{*}{ CHR } & \multirow[t]{2}{*}{ SNP } & \multirow[t]{2}{*}{ Position bp } & \multirow[t]{2}{*}{ Minor allele } & \multicolumn{4}{|c|}{ NELSON-COPD } & \multicolumn{4}{|c|}{ NELSON-non-COPD } & \multirow[t]{2}{*}{ Direction of effect ${ }^{\#}$} & \multirow[t]{2}{*}{ Closest gene(s) } \\
\hline & & & & MAF & Rank & $p$-value & OR & MAF & Rank & p-value & OR & & \\
\hline 1 & rs6677529 & 160530378 & $A$ & 0.19 & 48 & $7.24 \times 10^{-3}$ & 1.42 & 0.17 & 10 & $1.03 \times 10^{-3}$ & 1.45 & ++ & NOS1API \\
\hline 3 & rs12632852 & 11593682 & G & 0.40 & 2 & $3.20 \times 10^{-4}$ & 0.67 & 0.39 & 52 & $8.70 \times 10^{-3}$ & 1.28 & -+ & VGLL4" \\
\hline 3 & rs2574704 & 11630381 & G & 0.29 & 26 & $3.94 \times 10^{-3}$ & 0.72 & 0.29 & 4 & $5.25 \times 10^{-4}$ & 1.40 & -+ & VGLL4" \\
\hline 3 & rs2574720 & 11635412 & C & 0.26 & 7 & $1.08 \times 10^{-3}$ & 0.68 & 0.26 & 3 & $3.97 \times 10^{-4}$ & 1.43 & -+ & VGLL4" \\
\hline 3 & rs2616551 & 11642123 & G & 0.18 & 54 & $7.91 \times 10^{-3}$ & 0.69 & 0.18 & 2 & $3.57 \times 10^{-4}$ & 1.50 & -+ & VGLL4" \\
\hline 3 & rs12374151 & 16605508 & $A$ & 0.12 & 18 & $2.83 \times 10^{-3}$ & 0.61 & 0.13 & 48 & $7.25 \times 10^{-3}$ & 1.43 & -+ & $D A Z L^{\text {ๆ }}$ \\
\hline 3 & rs9852824 & 24397993 & $A$ & 0.46 & 50 & $7.51 \times 10^{-3}$ & 1.32 & 0.46 & 60 & $9.90 \times 10^{-3}$ & 0.79 & +- & $T H R B^{\pi}$ \\
\hline 3 & rs3796150 & 66584924 & $A$ & 0.20 & 55 & $8.54 \times 10^{-3}$ & 0.70 & 0.17 & 32 & $4.73 \times 10^{-3}$ & 0.70 & -- & $L R I G 1^{\Uparrow}$ \\
\hline 3 & rs7648171 & 106704936 & G & 0.20 & 41 & $6.16 \times 10^{-3}$ & 0.70 & 0.21 & 36 & $6.03 \times 10^{-3}$ & 0.73 & -- & ALCAMף \\
\hline 4 & rs4306981 & 80143145 & G & 0.31 & 1 & $4.40 \times 10^{-5}$ & 1.57 & 0.30 & 6 & $5.73 \times 10^{-4}$ & 1.40 & ++ & $P A Q R 3, A R D 1 B$ \\
\hline 4 & rs10518211 & 80156089 & G & 0.48 & 21 & $3.50 \times 10^{-3}$ & 1.35 & 0.48 & 20 & $1.93 \times 10^{-3}$ & 1.33 & ++ & $P A Q R 3, A R D 1 B$ \\
\hline 4 & rs4834752 & 120275247 & $A$ & 0.42 & 12 & $1.97 \times 10^{-3}$ & 0.72 & 0.44 & 15 & $1.30 \times 10^{-3}$ & 1.34 & -+ & MYOZ2 \\
\hline 4 & rs1017710 & 180937258 & A & 0.07 & 5 & $9.14 \times 10^{-4}$ & 1.97 & 0.07 & 37 & $6.23 \times 10^{-3}$ & 0.58 & +- & LOC391719, hCG_2025798 \\
\hline 4 & rs17068194 & 180952052 & $A$ & 0.07 & 6 & $9.14 \times 10^{-4}$ & 1.97 & 0.07 & 41 & $6.71 \times 10^{-3}$ & 0.58 & +- & LOC391719, hCG_2025798 \\
\hline 5 & rs365294 & 3476838 & $A$ & 0.38 & 45 & $6.74 \times 10^{-3}$ & 1.34 & 0.37 & 8 & $7.47 \times 10^{-4}$ & 1.38 & ++ & LOC100132531, IRX1 \\
\hline 5 & rs1995385 & 73415681 & G & 0.23 & 4 & $6.71 \times 10^{-4}$ & 0.65 & 0.23 & 58 & $9.39 \times 10^{-3}$ & 1.32 & -+ & RGNEF, ENC1 \\
\hline 5 & rs718164 & 73417137 & G & 0.23 & 3 & $5.37 \times 10^{-4}$ & 0.64 & 0.23 & 57 & $9.37 \times 10^{-3}$ & 1.32 & -+ & RGNEF, ENC2 \\
\hline 5 & rs11738681 & 176694141 & G & 0.33 & 43 & $6.35 \times 10^{-3}$ & 0.74 & 0.32 & 43 & $6.79 \times 10^{-3}$ & 0.76 & -- & LMAN2 \\
\hline 5 & rs11949401 & 176698595 & G & 0.33 & 36 & $5.26 \times 10^{-3}$ & 0.73 & 0.31 & 53 & $8.76 \times 10^{-3}$ & 0.76 & -- & LMAN2 \\
\hline 5 & rs9313758 & 176705697 & C & 0.33 & 44 & $6.35 \times 10^{-3}$ & 0.74 & 0.31 & 42 & $6.76 \times 10^{-3}$ & 0.76 & -- & LMAN2 $2 \pi$ \\
\hline 5 & rs4532376 & 176707009 & $A$ & 0.33 & 33 & $4.86 \times 10^{-3}$ & 0.73 & 0.31 & 33 & $5.13 \times 10^{-3}$ & 0.75 & -- & LMAN2 $2^{\Uparrow}$ \\
\hline 5 & rs4131289 & 176713151 & $A$ & 0.33 & 40 & $5.88 \times 10^{-3}$ & 0.74 & 0.31 & 29 & $4.15 \times 10^{-3}$ & 0.74 & -- & LMAN2, RGS14 \\
\hline 6 & rs10457138 & 106460454 & G & 0.27 & 15 & $2.47 \times 10^{-3}$ & 0.70 & 0.26 & 17 & $1.66 \times 10^{-3}$ & 1.37 & -+ & LOC100130683, PRDM1 \\
\hline 7 & rs40463 & 40915342 & $A$ & 0.12 & 24 & $3.65 \times 10^{-3}$ & 1.55 & 0.13 & 51 & $8.30 \times 10^{-3}$ & 0.68 & +- & C7orf10, INHBA \\
\hline 7 & rs4729686 & 100747270 & $A$ & 0.07 & 13 & $2.18 \times 10^{-3}$ & 0.50 & 0.07 & 22 & $2.76 \times 10^{-3}$ & 1.67 & -+ & $R A B L 5^{\natural}$ \\
\hline 7 & rs2905286 & 112081312 & G & 0.48 & 57 & $9.04 \times 10^{-3}$ & 0.76 & 0.48 & 39 & $6.56 \times 10^{-3}$ & 0.78 & -- & NPM1P14, LOC100128875 \\
\hline 8 & rs2055516 & 769714 & C & 0.25 & 11 & $1.85 \times 10^{-3}$ & 1.46 & 0.25 & 14 & $1.27 \times 10^{-3}$ & 1.40 & ++ & c8orf68 \\
\hline 8 & rs10105558 & 783149 & $A$ & 0.25 & 27 & $4.04 \times 10^{-3}$ & 1.42 & 0.25 & 28 & $3.65 \times 10^{-3}$ & 1.35 & ++ & C8orf68 \\
\hline 8 & rs13282923 & 4473969 & G & 0.29 & 29 & $4.10 \times 10^{-3}$ & 1.38 & 0.29 & 18 & $1.82 \times 10^{-3}$ & 0.72 & +- & CSMD1ๆ \\
\hline 8 & rs13273819 & 135514435 & $A$ & 0.23 & 35 & $5.25 \times 10^{-3}$ & 1.39 & 0.23 & 54 & $9.15 \times 10^{-3}$ & 1.32 & ++ & LOC100129104, ZFAT \\
\hline 9 & rs530582 & 134354849 & G & 0.15 & 17 & $2.76 \times 10^{-3}$ & 0.64 & 0.17 & 7 & $6.63 \times 10^{-4}$ & 1.49 & -+ & $R P 11-738114.8^{9}$ \\
\hline 10 & rs10903396 & 1208030 & G & 0.46 & 28 & $4.06 \times 10^{-3}$ & 0.74 & 0.46 & 38 & $6.26 \times 10^{-3}$ & 0.78 & -- & C10orf139, LOC100130729 \\
\hline 10 & rs10905113 & 7246430 & G & 0.44 & 8 & $1.14 \times 10^{-3}$ & 1.41 & 0.44 & 50 & $8.12 \times 10^{-3}$ & 0.79 & +- & SFMBT2ף \\
\hline 10 & rs17601717 & 52831431 & G & 0.23 & 39 & $5.38 \times 10^{-3}$ & 0.71 & 0.25 & 40 & $6.57 \times 10^{-3}$ & 1.32 & -+ & $P R K G 1^{\Uparrow}$ \\
\hline 10 & rs7902476 & 72693742 & $A$ & 0.11 & 25 & $3.70 \times 10^{-3}$ & 0.60 & 0.12 & 26 & $3.37 \times 10^{-3}$ & 0.64 & -- & UNC5Bף \\
\hline 11 & rs2273688 & 35295319 & $A$ & 0.27 & 31 & $4.49 \times 10^{-3}$ & 0.71 & 0.28 & 16 & $1.56 \times 10^{-3}$ & 1.40 & -+ & SLC1A2 \\
\hline 11 & rs10768129 & 35319065 & $A$ & 0.27 & 47 & $7.02 \times 10^{-3}$ & 0.72 & 0.28 & 13 & $1.21 \times 10^{-3}$ & 1.40 & -+ & SLC1A2 \\
\hline 11 & rs7127824 & 35330427 & $A$ & 0.27 & 22 & $3.64 \times 10^{-3}$ & 0.70 & 0.28 & 11 & $1.14 \times 10^{-3}$ & 1.40 & -+ & SLC1A2 \\
\hline 11 & rs7130967 & 35330584 & $A$ & 0.27 & 23 & $3.64 \times 10^{-3}$ & 0.70 & 0.28 & 12 & $1.14 \times 10^{-3}$ & 1.40 & -+ & SLC1A2 \\
\hline 11 & rs927352 & 35334090 & $A$ & 0.30 & 58 & $9.36 \times 10^{-3}$ & 0.73 & 0.31 & 19 & $1.90 \times 10^{-3}$ & 1.36 & -+ & SLC1A2 \\
\hline 11 & rs11033910 & 37021958 & G & 0.28 & 53 & $7.82 \times 10^{-3}$ & 0.73 & 0.29 & 56 & $9.32 \times 10^{-3}$ & 1.30 & -+ & C11orf74, LOC100129825 \\
\hline 11 & rs12417575 & 85832165 & G & 0.28 & 37 & $5.31 \times 10^{-3}$ & 0.72 & 0.27 & 59 & $9.85 \times 10^{-3}$ & 0.76 & -- & $M E 3^{\text {भी }}$ \\
\hline 11 & rs689051 & 124797700 & $A$ & 0.16 & 10 & $1.43 \times 10^{-3}$ & 1.58 & 0.15 & 30 & $4.40 \times 10^{-3}$ & 0.67 & +- & PKNOX2 \\
\hline
\end{tabular}




\begin{tabular}{|c|c|c|c|c|c|c|c|c|c|c|c|c|c|}
\hline \multirow[t]{2}{*}{ CHR } & \multirow[t]{2}{*}{ SNP } & \multirow[t]{2}{*}{ Position bp } & \multirow[t]{2}{*}{ Minor allele } & \multicolumn{4}{|c|}{ NELSON-COPD } & \multicolumn{4}{|c|}{ NELSON-non-COPD } & \multirow[t]{2}{*}{ Direction of effect ${ }^{\#}$} & \multirow[t]{2}{*}{ Closest gene(s) } \\
\hline & & & & MAF & Rank & p-value & OR & MAF & Rank & p-value & OR & & \\
\hline 12 & rs17179798 & 5184769 & $A$ & 0.24 & 52 & $7.73 \times 10^{-3}$ & 1.38 & 0.23 & 27 & $3.51 \times 10^{-3}$ & 1.37 & ++ & KCNA5, LOC387826 \\
\hline 12 & rs1894307 & 11896987 & $A$ & 0.15 & 34 & $4.90 \times 10^{-3}$ & 1.49 & 0.14 & 9 & $9.39 \times 10^{-4}$ & 1.50 & ++ & ETV6" \\
\hline 12 & rs2255953 & 11902003 & $G$ & 0.23 & 59 & $9.78 \times 10^{-3}$ & 1.38 & 0.21 & 5 & $5.34 \times 10^{-4}$ & 1.45 & ++ & ETV6" \\
\hline 12 & rs2855708 & 11904839 & G & 0.28 & 30 & $4.10 \times 10^{-3}$ & 1.40 & 0.27 & 34 & $5.40 \times 10^{-3}$ & 1.31 & ++ & ETV6" \\
\hline 12 & rs1820545 & 39096860 & G & 0.41 & 38 & $5.32 \times 10^{-3}$ & 0.75 & 0.42 & 31 & $4.47 \times 10^{-3}$ & 1.29 & -+ & LRRK2, MUC19 \\
\hline 12 & rs7306163 & 39111184 & C & 0.41 & 42 & $6.21 \times 10^{-3}$ & 0.75 & 0.42 & 35 & $5.50 \times 10^{-3}$ & 1.28 & -+ & MUC19ף \\
\hline 14 & rs8009673 & 31412453 & A & 0.14 & 46 & $7.00 \times 10^{-3}$ & 1.50 & 0.13 & 21 & $2.23 \times 10^{-3}$ & 1.49 & ++ & NUBPL, C14orf128 \\
\hline 14 & rs7155416 & 76021126 & $A$ & 0.12 & 51 & $7.72 \times 10^{-3}$ & 1.51 & 0.14 & 23 & $3.02 \times 10^{-3}$ & 1.46 & ++ & ESRRB \\
\hline 14 & rs9323838 & 88789353 & G & 0.37 & 56 & $8.68 \times 10^{-3}$ & 1.33 & 0.38 & 49 & $7.94 \times 10^{-3}$ & 0.78 & +- & FOXN3" \\
\hline 15 & rs1531636 & 92404552 & $A$ & 0.36 & 14 & $2.36 \times 10^{-3}$ & 1.40 & 0.34 & 44 & $7.05 \times 10^{-3}$ & 1.28 & ++ & LOC283682, LOC100129642 \\
\hline 16 & rs7202333 & 67438996 & $G$ & 0.39 & 32 & $4.76 \times 10^{-3}$ & 0.73 & 0.37 & 47 & $7.24 \times 10^{-3}$ & 0.77 & -- & TMCO \\
\hline 16 & rs7184633 & 81379514 & $A$ & 0.40 & 19 & $2.93 \times 10^{-3}$ & 0.73 & 0.40 & 1 & $2.67 \times 10^{-4}$ & 0.71 & -- & $\mathrm{CDH} 13^{\pi}$ \\
\hline 19 & rs10411733 & 62482800 & A & 0.47 & 16 & $2.60 \times 10^{-3}$ & 0.73 & 0.46 & 25 & $3.29 \times 10^{-3}$ & 1.31 & -+ & ZNF460" \\
\hline 20 & rs2224326 & 19689491 & A & 0.23 & 9 & $1.31 \times 10^{-3}$ & 0.66 & 0.24 & 46 & $7.15 \times 10^{-3}$ & 1.31 & -+ & LOC100130408" \\
\hline 20 & rs4811610 & 53652782 & $G$ & 0.29 & 60 & $9.92 \times 10^{-3}$ & 1.33 & 0.31 & 45 & $7.11 \times 10^{-3}$ & 0.76 & +- & RPL12P4, CBLN4 \\
\hline 22 & rs2073760 & 17886456 & $A$ & 0.40 & 49 & $7.33 \times 10^{-3}$ & 1.32 & 0.40 & 24 & $3.20 \times 10^{-3}$ & 0.76 & +- & $C D C 45 L^{\text {ๆ }}$ \\
\hline 22 & rs467768 & 28291986 & $A$ & 0.14 & 20 & $3.43 \times 10^{-3}$ & 0.64 & 0.15 & 55 & $9.29 \times 10^{-3}$ & 0.70 & -- & NIPSNAP1" \\
\hline
\end{tabular}

CHR: chromosome; MAF: minor allele frequency. ${ }^{\#}$ : in the order NELSON-COPD and NELSON-non-COPD, where - indicates odds ratio $\leqslant 0.95$ and + indicates odds ratio $>1.05 ;{ }^{\uparrow}:$ SNP present in intron. 
The specificity of these functions is regulated by protein-protein interactions [31]. SOX proteins also regulate the Wnt signalling pathway required for the specification and differentiation of lung epithelial cells, by interacting with $\beta$-catenin [31]. As SOX1 and MAML3 are both associated with $\beta$-catenin, it is conceivable that there is a link between these genes and $\mathrm{CMH}$.

There are limitations to the study. We did not have post-bronchodilator spirometry data; therefore, some individuals without COPD may have been in advertently included in the COPD group. The power of each identification analysis (338 cases and 511 controls with COPD, and 342 cases and 1006 controls without COPD) is rather limited, possibly explaining the lack of genome-wide significant findings. Moreover, some replication cohorts were underpowered and $\mathrm{CMH}$ is rather a rough estimate. However, we found suggestive evidence of a genetic contribution to $\mathrm{CMH}$ in the full population without stratification for COPD, thus suggesting that power would be more of a problem than the definition of $\mathrm{CMH}$ [14]. When we analysed whether our previously reported gene SATB1 was associated with $\mathrm{CMH}$ in individuals with and without COPD, we also found that the significance was considerably reduced, p-values of rs6577641 being $2.52 \times 10^{-2}$ and $5.69 \times 10^{-2}$, respectively.

In summary, we found no significant overlap between genes associated with $\mathrm{CMH}$ in individuals with COPD and without COPD. In COPD, lower GDNF mRNA expression in bronchial biopsies was significantly associated with $\mathrm{CMH}$, possibly by the altered action of GDNF-AS1, our top gene. Furthermore, in individuals without COPD, a top SNP in MAML3 that was nominally replicated in the non-COPD cohort was an eQTL in lung tissue. Our results suggest genetic heterogeneity of $\mathrm{CMH}$ in individuals with and without COPD, and indicate that it is worthwhile to repeat this study in much larger cohorts.

\section{Acknowledgements}

The authors affiliations are as follows. A.E. Dijkstra: University of Groningen, University Medical Center Groningen, Dept of Pulmonology and GRIAC Research Institute, Groningen, The Netherlands; H.M. Boezen: University of Groningen, University Medical Center Groningen, GRIAC Research Institute and Dept of Epidemiology, Groningen, The Netherlands; M. van den Berge: University of Groningen, University Medical Center Groningen, Dept of Pulmonology and GRIAC Research Institute, Groningen, The Netherlands; J.M. Vonk: University of Groningen, University Medical Center Groningen, GRIAC Research Institute and Dept of Epidemiology, Groningen, The Netherlands; P.S. Hiemstra: Dept of Pulmonology, Leiden University Medical Center, Leiden, The Netherlands; R.G. Barr: Dept of Medicine, College of Physicians and Surgeons, and Dept of Epidemiology, Mailman School of Public Health, Columbia University, New York, NY, USA; K.M. Burkart: Dept of Medicine, College of Physicians and Surgeons, Columbia University, New York, NY, USA; A. Manichaikul: Center for Public Health Genomics and Dept of Public Health Sciences, Division of Biostatistics and Epidemiology, University of Virginia, Charlottesville, VA, USA; T.D. Pottinger: Dept of Medicine, College of Physicians and Surgeons, Columbia University, New York, NY, USA; E.K. Silverman: Channing Division of Network Medicine, Dept of Medicine, and Division of Pulmonary and Critical Care Medicine, Dept of Medicine, Brigham and Women's Hospital, and Harvard Medical School, Boston, MA, USA; M.H. Cho: Channing Division of Network Medicine, Dept of Medicine, and Division of Pulmonary and Critical Care Medicine, Dept of Medicine, Brigham and Women's Hospital, and Harvard Medical School, Boston, MA, USA; J.D. Crapo: Division of Pulmonary and Critical Care Medicine, National Jewish Health, Denver, CO, USA; T.H. Beaty: Dept of Epidemiology, Johns Hopkins Bloomberg School of Public Health, Baltimore, MD, USA; Per Bakke: Dept of Thoracic Medicine, Haukeland University, Hospital and Dept of Clinical Science, University of Bergen, Bergen, Norway; A. Gulsvik: Dept of Thoracic Medicine, Haukeland University, Hospital and Dept of Clinical Science, University of Bergen, Bergen, Norway; D.A. Lomas: Wolfson Institute for Biomedical Research, University College London, London, UK; Y. Bossé: Institut Universitaire de Cardiologie et de Pneumologie de Québec, Dept of Molecular Medicine, Laval University, Québec City, QC, Canada; D.C. Nickle: Merck Research Laboratories, Boston, MA, USA; P.D. Paré: Division of Respirology, Dept of Medicine, Center for Heart Lung Innovation, St Paul's Hospital, University of British Columbia, Vancouver, BC, Canada; H.J. de Koning: Dept of Public Health, Erasmus Medical Center Rotterdam, Rotterdam, The Netherlands; J-W. Lammers: Dept of Pulmonology, University Medical Center Utrecht, Utrecht, The Netherlands; P. Zanen: Dept of Pulmonology, University Medical Center Utrecht, Utrecht, The Netherlands; J. Smolonska: University of Groningen, University Medical Center Groningen, GRIAC Research Institute and Dept of Genetics, Groningen, The Netherlands; C. Wijmenga: University of Groningen, University Medical Center Groningen, Dept of Genetics, Groningen, The Netherlands; C-A. Brandsma: University of Groningen, University Medical Center Groningen, GRIAC Research Institute, and Dept of Pathology and Medical Biology, Groningen, The Netherlands; H.J.M. Groen: University of Groningen, University Medical Center Groningen, Dept of Pulmonology, Groningen, The Netherlands; D.S. Postma: University of Groningen, University Medical Center Groningen, Dept of Pulmonology and GRIAC Research Institute, Groningen, The Netherlands.

The members of the LifeLines Cohort Study group are: B.Z. Alizadeh (University of Groningen, University Medical Center Groningen, Dept of Epidemiology, Groningen, the Netherlands), R.A. de Boer (University of Groningen, University Medical Center Groningen, Dept of Cardiology, Groningen, the Netherlands), H.M. Boezen, M. Bruinenberg (University of Groningen, University Medical Center Groningen, the LifeLines Cohort Study, Groningen, the Netherlands), L. Franke (University of Groningen, University Medical Center Groningen, Dept of Genetics, Groningen, the Netherlands), P. van der Harst (University of Groningen, University Medical Center Groningen, Department of Cardiology, Groningen, the Netherlands), H.L. Hillege (University of Groningen, University Medical Center Groningen, Depts of Epidemiology and Cardiology, Groningen, the Netherlands), M.M. van der Klauw (University of Groningen, University Medical Center Groningen, Dept of Endocrinology, Groningen, the Netherlands), G. Navis (University of Groningen, University Medical Center Groningen, Dept of Internal Medicine, Division of Nephrology, Groningen, the Netherlands), J. Ormel (University of Groningen, University Medical Center Groningen, Interdisciplinary Center of 
Psychopathology of Emotion Regulation (ICPE), Dept of Psychiatry, Groningen, the Netherlands), D.S. Postma, J.G.M. Rosmalen (University of Groningen, University Medical Center Groningen, ICPE, Dept of Psychiatry, Groningen, the Netherlands), J.P. Slaets (University of Groningen, University Medical Center Groningen, Depts of Internal Medicine and Geriatrics, Groningen, the Netherlands), H. Snieder (University of Groningen, University Medical Center Groningen, Dept of Epidemiology, Groningen, the Netherlands), R.P. Stolk (University of Groningen, University Medical Center Groningen, Dept of Epidemiology, Groningen, the Netherlands), B.H.R. Wolffenbuttel (University of Groningen, University Medical Center Groningen, Dept of Endocrinology, Groningen, the Netherlands) and C. Wijmenga (University of Groningen, University Medical Center Groningen, Dept of Genetics, Groningen, the Netherlands)

The authors would like to thank the staff at the Respiratory Health Network Tissue Bank of the FRQS for their valuable assistance.

The authors would like to thank the COPDGene investigators at the following Core Units. Administrative Core: J.D. Crapo (principal investigator), E.K. Silverman (principal investigator), B. Make, E.A. Regan, S. Penchev, R. Lantz, S. Melanson and L. Stepp. Genetic Analysis Core: T. Beaty, N. Laird, C. Lange, M. Cho, S. Santorico, J. Hokanson, D. DeMeo, N. Hansel, C. Hersh, P. Castaldi, M-L. McDonald, J. Zhou, M. Mattheisen, E. Wan, M. Hardin, J. Hetmanski, M. Parker and T. Murray. Imaging Core: D. Lynch, J. Schroeder, J. Newell Jr, J. Reilly, H. Coxson, P. Judy, E. Hoffman, G. Washko, R. San Jose Estepar, J. Ross, M. Al Qaisi, J. Zach, A. Kluiber, J. Sieren, T. Mann, D. Richert, A. McKenzie, J. Akhavan and D. Stinson. PFT QA Core, National Jewish Health: R. Jensen. Biological Repository, Johns Hopkins University, Baltimore, MD, USA: H. Farzadegan, S. Meyerer, S. Chandan and Samantha Bragan. Data Coordinating Center and Biostatistics, National Jewish Health: D. Everett, A. Williams, C. Wilson, A. Forssen, A. Powell and J. Piccoli. Epidemiology Core, University of Colorado School of Public Health, Denver, CO, USA: J. Hokanson, M. Sontag, J. Black-Shinn, G. Kinney and S. Lutz.

The authors would like to thank the COPDGene investigators at the following Clinical Centers. Ann Arbor Veterans Affairs (VA), Ann Arbor, MI, USA: J. Curtis and E. Kazerooni. Baylor College of Medicine, Houston, TX, USA: N. Hanania, P. Alapat, V. Bandi, K. Guntupalli, E. Guy, A. Mallampalli, C. Trinh, M. Atik, H. Al-Azzawi, M. Willis, S. Pinero, L. Fahr, A. Nachiappan, C. Bray, L.A. Frigini, C. Farinas, D. Katz, J. Freytes and A.M. Marciel. Brigham and Women's Hospital: D. DeMeo, C. Hersh, G. Washko, F. Jacobson, H. Hatabu, P. Clarke, R. Gill, A. Hunsaker, B. Trotman-Dickenson and R. Madan. Columbia University: R.G. Barr, B. Thomashow, J. Austin and B. D'Souza. Duke University Medical Center, Durham, NC, USA: N. MacIntyre Jr, L. Washington and H.P. McAdams. Reliant Medical Group, Worcester, MA, USA: R. Rosiello, T. Bresnahan, J. Bradley, S. Kuong, S. Meller and S. Roland. Health Partners Research Foundation, Minneapolis, MN, USA: C. McEvoy and J. Tashjian. Johns Hopkins University: R. Wise, N. Hansel, R. Brown, G. Diette and K. Horton. Los Angeles Biomedical Research Institute at Harbor UCLA Medical Center, Torrance, CA, USA: R. Casaburi, J. Porszasz, H. Fischer and M. Budoff. Michael E. DeBakey Veterans Affairs Medical Center, Houston, TX, USA: A. Sharafkhaneh, C. Trinh, H. Kamal, R. Darvishi, M. Willis, S. Pinero, L. Fahr, A. Nachiappan, C. Bray, L.A. Frigini, C. Farinas, D. Katz, J. Freytes and A.M. Marciel. Minneapolis VA, Minneapolis, MN, USA: D. Niewoehner, Q. Anderson, K. Rice and A. Caine. Morehouse School of Medicine, Atlanta, GA, USA: M. Foreman, G. Westney and E. B. National Jewish Health: R. Bowler, D. Lynch, J. Schroeder, V. Hale, J. Armstrong II, D. Dyer, J. Chung and C. Cox. Temple University, Philadelphia, PA, USA: G. Criner, V. Kim, N. Marchetti, A. Satti, A.J. Mamary, R. Steiner, C. Dass and L. Cone. University of Alabama, Birmingham, AL, USA: W. Bailey, M. Dransfield, M. Wells, S. Bhatt, H. Nath and S. Singh. University of California, San Diego, CA, USA: J. Ramsdell and P. Friedman. University of Iowa, Iowa City, IA, USA: A. Cornellas, J. Newell Jr and E.J.R. van Beek. University of Michigan, Ann Arbor, MI, USA: F. Martinez, M. Han and E. Kazerooni. University of Minnesota, Minneapolis, MN, USA: C. Wendt and T. Allen. University of Pittsburgh, Pittsburgh, PA, USA: F. Sciurba, J. Weissfeld, C. Fuhrman, J. Bon and D. Hooper. University of Texas Health Science Center at San Antonio, San Antonio, TX, USA: A. Anzueto, S. Adams, C. Orozco, M. Ruiz, A. Mumbower, A. Kruger, C. Restrepo and M. Lane.

The principal investigators and centres participating in ECLIPSE were as follows. Bulgaria: Y. Ivanov, Pleven; K. Kostov, Sofia. Canada: J. Bourbeau, Montreal, QC; M. Fitzgerald, Vancouver, BC; P. Hernández, Halifax, NA; K. Killian, Hamilton, ON; R. Levy, Vancouver, BC; F. Maltais, Montreal, QC; D. O’Donnell, Kingston, ON. Czech Republic: J. Krepelka, Prague. Denmark: J. Vestbo, Hvidovre. The Netherlands: E. Wouters, Horn. New Zealand: D. Quinn, Wellington. Norway: P. Bakke, Bergen; Slovenia: M. Kosnik, Golnik. Spain: A. Agusti and Jaume Sauleda, Palma de Mallorca. Ukraine: Y. Feschenko, V. Gavrisyuk and L. Yashina, Kiev. UK: W. MacNee, Edinburgh; D. Singh, Manchester; J. Wedzicha, London. USA: A. Anzueto, San Antonio, TX; S. Braman, Providence, RI; R. Casaburi, Torrance, CA; B. Celli, Boston, MA; G. Giessel, Richmond, VA; M. Gotfried, Phoenix, AZ; G. Greenwald, Rancho Mirage, CA; N. Hanania, Houston, TX; D. Mahler, Lebanon, NH; B. Make, Denver, CO; S. Rennard, Omaha, NE; C. Rochester, New Haven, CT; P. Scanlon, Rochester, MN; D. Schuller, Omaha, NE; F. Sciurba, Pittsburgh, PA; A. Sharafkhaneh, Houston, TX; T. Siler, St Charles, MO; E. Silverman, Boston, MA; A. Wanner, Miami, FL; R. Wise, Baltimore, MD; R. ZuWallack, Hartford, CT. Steering Committee: H. Coxson (Canada), C. Crim (GlaxoSmithKline, USA), L. Edwards (GlaxoSmithKline, USA), D. Lomas (UK), W. MacNee (UK), E. Silverman (USA), R. Tal Singer (co-chair; GlaxoSmithKline, USA), J. Vestbo (co-chair, Denmark), J. Yates (GlaxoSmithKline, USA). Scientific Committee: A. Agusti (Spain), P. Calverley (UK), B. Celli (USA), C. Crim (GlaxoSmithKline, USA), B. Miller (GlaxoSmithKline, USA), W. MacNee (Chair, UK), S. Rennard (USA), R. Tal-Singer (GlaxoSmithKline, USA), E. Wouters (The Netherlands), J. Yates (GlaxoSmithKline, USA).

\section{References}

1 de Oca MM, Halbert RJ, Lopez MV, et al. The chronic bronchitis phenotype in subjects with and without COPD: the PLATINO study. Eur Respir J 2012; 40: 28-36.

2 Kim V, Criner GJ. Chronic bronchitis and chronic obstructive pulmonary disease. Am J Respir Crit Care Med 2013; 187: 228-237.

3 Vestbo J. Epidemiological studies in mucus hypersecretion. Novartis Found Symp 2002; 248: 3-12.

4 Agusti A, Calverley PM, Celli B, et al. Characterisation of COPD heterogeneity in the ECLIPSE cohort. Respir Res 2010; 11: 122 
5 Dijkstra AE, de Jong K, Boezen HM, et al. Risk factors for chronic mucus hypersecretion in individuals with and without COPD: influence of smoking and job exposure on CMH. Occup Environ Med 2014; 71: 346-352.

6 Lange P, Parner J, Prescott E, et al. Chronic bronchitis in an elderly population. Age Ageing 2003; 32: 636-642.

7 Patel IS, Seemungal TA, Wilks M, et al. Relationship between bacterial colonisation and the frequency, character, and severity of COPD exacerbations. Thorax 2002; 57: 759-764.

8 Vestbo J. Chronic bronchitis: should it worry us? Chron Respir Dis 2004; 1: 173-176.

9 Forey BA, Thornton AJ, Lee PN. Systematic review with meta-analysis of the epidemiological evidence relating smoking to COPD, chronic bronchitis and emphysema. BMC Pulm Med 2011; 11: 36

10 Viegi G, Carrozzi L, Di Pede F, et al. Risk factors for chronic obstructive pulmonary disease in a north Italian rural area. Eur J Epidemiol 1994; 10: 725-731.

11 Silverman EK, Chapman HA, Drazen JM, et al. Genetic epidemiology of severe, early-onset chronic obstructive pulmonary disease. Risk to relatives for airflow obstruction and chronic bronchitis. Am J Respir Crit Care Med 1998; 157: 1770-1778.

12 Hallberg J, Dominicus A, Eriksson UK, et al. Interaction between smoking and genetic factors in the development of chronic bronchitis. Am J Respir Crit Care Med 2008; 177: 486-490.

13 Zhu G, Agusti A, Gulsvik A, et al. CTLA4 gene polymorphisms are associated with chronic bronchitis. Eur Respir J 2009; 34: 598-604.

14 Dijkstra AE, Smolonska J, van den Berge M, et al. Susceptibility to chronic mucus hypersecretion, a genome wide association study. PLoS One 2014; 9: e91621

15 van Klaveren RJ, Oudkerk M, Prokop M, et al. Management of lung nodules detected by volume CT scanning. $N$ Engl J Med 2009; 361: 2221-2229.

16 Quanjer PH, Tammeling GJ, Cotes JE, et al. Lung volumes and forced ventilatory flows. Report Working Party Standardization of Lung Function Tests, European Community for Steel and Coal. Official Statement of the European Respiratory Society. Eur Respir J 1993; 6: Suppl. 16, 5-40.

17 Cho MH, Castaldi PJ, Wan ES, et al. A genome-wide association study of COPD identifies a susceptibility locus on chromosome 19q13. Hum Mol Genet 2012; 21: 947-957.

18 Regan EA, Hokanson JE, Murphy JR, et al. Genetic epidemiology of COPD (COPDGene) study design. COPD 2010; 7: 32-43.

19 Vestbo J, Anderson W, Coxson HO, et al. Evaluation of COPD Longitudinally to Identify Predictive Surrogate End-points (ECLIPSE). Eur Respir J 2008; 31: 869-873.

20 Bild DE, Bluemke DA, Burke GL, et al. Multi-ethnic study of atherosclerosis: objectives and design. Am J Epidemiol 2002; 156: 871-881.

21 Hao K, Bosse Y, Nickle DC, et al. Lung eQTLs to help reveal the molecular underpinnings of asthma. PLoS Genet 2012; 8: e1003029

22 Lapperre TS, Snoeck-Stroband JB, Gosman MM, et al. Effect of fluticasone with and without salmeterol on pulmonary outcomes in chronic obstructive pulmonary disease: a randomized trial. Ann Intern Med 2009; 151: 517-527.

23 van den Berge M, Steiling K, Timens W, et al. Airway gene expression in COPD is dynamic with inhaled corticosteroid treatment and reflects biological pathways associated with disease activity. Thorax 2014; 69: 14-23.

24 Purcell S, Neale B, Todd-Brown K, et al. PLINK: a tool set for whole-genome association and population-based linkage analyses. Am J Hum Genet 2007; 81: 559-575.

25 Weiss B, Davidkova G, Zhou LW. Antisense RNA gene therapy for studying and modulating biological processes. Cell Mol Life Sci 1999; 55: 334-358.

26 Tollet J, Everett AW, Sparrow MP. Development of neural tissue and airway smooth muscle in fetal mouse lung explants: a role for glial-derived neurotrophic factor in lung innervation. Am J Respir Cell Mol Biol 2002; 26: 420-429.

27 Karihaloo A, Nickel C, Cantley LG. Signals which build a tubule. Nephron Exp Nephrol 2005; 100: e40-e45.

28 Lieu TM, Myers AC, Meeker S, et al. TRPV1 induction in airway vagal low-threshold mechanosensory neurons by allergen challenge and neurotrophic factors. Am J Physiol Lung Cell Mol Physiol 2012; 302: L941-L948.

29 Oyama T, Harigaya K, Sasaki N, et al. Mastermind-like 1 (MamL1) and mastermind-like 3 (MamL3) are essential for Notch signaling in vivo. Development 2011; 138: 5235-5246.

30 van Es JH, van Gijn ME, Riccio O, et al. Notch/gamma-secretase inhibition turns proliferative cells in intestinal crypts and adenomas into goblet cells. Nature 2005; 435: 959-963.

31 Bernard P, Harley VR. Acquisition of SOX transcription factor specificity through protein-protein interaction, modulation of Wnt signalling and post-translational modification. Int J Biochem Cell Biol 2010; 42: 400-410. 